\title{
Israel Moiseevich Gelfand, Part I
}

\section{Vladimir Retakh, Coordinating Editor}

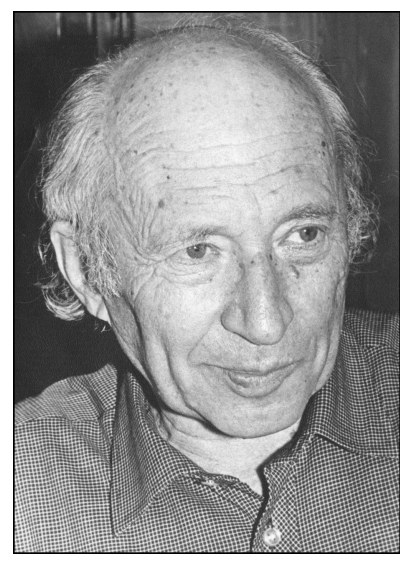

I. M. Gelfand
Israel Moiseevich Gelfand, a mathematician compared by Henri Cartan to Poincaré and Hilbert, was born on September 2,1913 , in the small town of Okny (later Red Okny) near Odessa in the Ukraine and died in New Brunswick, New Jersey, USA, on October 5, 2009.

Nobody guided Gelfand in his studies. He attended the only school in town, and his mathematics teacher could offer him nothing except encouragement-and this was very important. In Gelfand's own words: "Offering encouragement is a teacher's most important job." In 1923 the family moved to another place and Gelfand entered a vocational school for chemistry lab technicians. However, he was expelled in the ninth grade as a son of a "bourgeois element" ("netrudovoi element" in Soviet parlance)-his father was a mill manager. After that Gelfand (he was sixteen and a half at that time) decided to go to Moscow, where he had some distant relatives.

Until his move to Moscow in 1930, Gelfand lived in total mathematical isolation. The only books available to him were secondary school texts and several community college textbooks. The most advanced of these books claimed that there are three kinds of functions: analytical, defined by formulas; empirical, defined by tables; and correlational. Like Ramanujan, he was experimenting a lot. Around

Vladimir Retakh is professor of mathematics at Rutgers University. His email address is vretakh@math. rutgers. edu.

He thanks Mark Saul for his help in the preparation of this collection.

The AMS wants to thank M. E. Bronstein, Elena Ermakova, Tatiana I. Gelfand, Tatiana V. Gelfand, and Carol Tate for their help with photographs for this article.

DOI: http://dx.doi.org/10.1090/noti937 age twelve Gelfand understood that some problems in geometry cannot be solved algebraically and drew a table of ratios of the length of the chord to the length of the arc. Much later it became clear for him that in fact he was drawing trigonometric tables.

From this period came his Mozartean style and his belief in the unity and harmony of mathematics (including applied mathematics) the unity determined not by rigid and loudly proclaimed programs, but rather by invisible and sometimes hidden ties connecting seemingly different areas. Gelfand described his school years and mathematical studies in an interview published in Quantum, a science magazine for high school students [1]. In Gelfand's own words: "It is my deep conviction that mathematical ability in most future professional mathematicians appears...when they are 13 to 16 years old.... This period formed my style of doing mathematics. I studied different subjects, but the artistic form of mathematics that took root at this time became the basis of my taste in choosing problems that continue to attract me to this day. Without understanding this motivation, I think it is impossible to make heads or tails of the seeming illogicality of my ways in working and the choice of themes in my work. Because of this motivating force, however, they actually come together sequentially and logically."

The interview also shows how a small provincial boy was jumping over centuries in his mathematical discoveries. At the age of fifteen Gelfand learned of a series for calculating the sine. He described this moment in the "Quantum" interview: "Before this I thought there were two types of mathematics, algebraic and geometric... When I discovered that the sine can be expressed algebraically as a series, the barriers came tumbling down, and mathematics became one. To this day I see various branches of mathematics, together with mathematical physics, as parts of a united whole."

After arriving in Moscow, Gelfand did not have steady work and lived on earnings from 
occasional odd jobs. At some point he had the good fortune to work at the checkout counter at the State (Lenin's) Library. This gave Gelfand a rare opportunity to talk with mathematics students from Moscow University. He also started to attend university seminars, where he found himself under intense psychological stress: new breezes were blowing in mathematics with the new demands for rigorous proofs. It was so different from his "homemade" experiments and his romantic views of mathematics. He also learned that none of his discoveries were new. Neither this nor other circumstances of his life deterred him, and his interest in mathematics continued to grow.

Just as his abrupt expulsion from school, the next twist in Gelfand's fate was also one of many paradoxes of life in the Soviet Union. On one hand, as a son of a "bourgeois element" he could not be a university student. On the other hand, at eighteen he was able to obtain a teaching position at one of many newly created technical colleges and at nineteen to enter the Ph.D. program at Moscow University. The reasons were simple: the Soviet state needed knowledgeable instructors to educate its future engineers and scientists of the proper "proletarian origin". But at that time the system was not rigid enough to purge or even strictly regulate graduate schools. As a result, a talented boy was able to enter a Ph.D. program without a college or even a high school diploma.

At the beginning of his career Gelfand was influenced by several Moscow mathematicians, especially his thesis adviser, A. N. Kolmogorov. In the Quantum interview Gelfand said that from Kolmogorov he learned "that a true mathematician must be a philosopher of nature." Another influence was the brilliant L. G. Shnirelman. In 1935 Gelfand defended his "candidate" (Ph.D.) thesis and in 1940 obtained the higher degree of Doctor of Science. In 1933 he began teaching at Moscow University, where he became a full professor in 1943 and started his influential seminar. He lost this position temporarily in 1952 during the infamous "anticosmopolitans" (in fact, anti-Semitic) campaign but was allowed to continue the seminar. Gelfand also worked at the Steklov Institute and for many years at the Institute for Applied Mathematics. There he took part in the secret program related to the Soviet version of the Manhattan Project and its extensions. Andrei Sakharov mentioned his work with Gelfand in [1].

In 1953 Gelfand was elected a Corresponding Member of the Academy of Science (an important title in the Soviet hierarchy). This happened right after Stalin's death and at the end of the anticosmopolitans campaign. According to Gelfand, the political uncertainty of the times made his election possible. Later the situation in the USSR stabilized, anti-Semitism became part of the Soviet system, and Gelfand became a full member of the Soviet Academy only in 1984 after being elected to leading foreign academies. In 1989 Gelfand moved to the United States. After spending some time at Harvard and MIT, he became a professor at Rutgers University, where he worked until his death.

The articles in the Notices present various descriptions of Gelfand's multifaceted research, his way of doing mathematics, and interaction with people. A leading American expert once told me that after reading all definitions in Gelfand's papers, he could easily prove all his theorems. Well, this easiness was based on long computations and a thorough consideration of a variety of carefully selected examples. Gelfand himself liked to repeat a statement by a Moscow mathematician: "Gelfand cannot prove hard theorems. He just turns any Gelfand at age 3, 1916. theorem into an easy one."

Gelfand always was surrounded by numerous collaborators attracted by his legendary intuition and the permanent flow of new ideas: in every decade he was establishing a new area of research. He had completely different approaches to different people, as described by A. Vershik, A. Zelevinsky, and me in our recollections. His students and collaborators represent an unusual variety of styles and interests. The only similarity for members of the Gelfand school was their inherited passion for mathematics.

One cannot write about Gelfand without mentioning his legendary seminar, which started in 1943 and continued until his death. Gelfand considered the seminar one of his most important creations. It is hard to describe the seminar in a few words: it was a "mathematical stock exchange", a breeding ground for young scientists, a demonstration of how to think about mathematics, a one-man show, and much more. It was not about functional analysis or geometry, it was about mathematics. Some would come to the seminar just to hear Gelfand's jokes and paradoxes. For example, after his visit to the U.S. he stated, "[The] Mathematical 


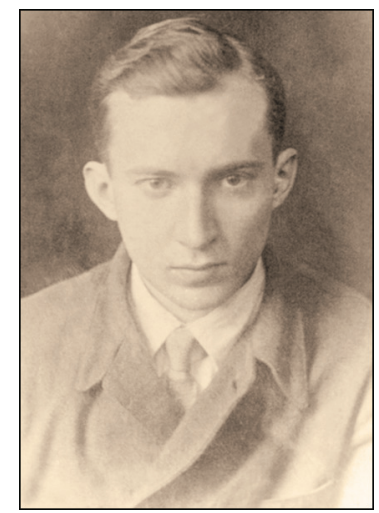

world is not a metric space: The distance from Harvard to MIT is greater than the sum of distances from Harvard to Moscow and from Moscow to MIT."

One should add that sometimes Gelfand's jokes were rather sharp, but for a young person to become a subject of Gelfand's joke meant to be noticed, to be knighted. The seminar was also the right place to find out about fresh preprints coming from the inaccessible West. But

Gelfand in 1934. most participants were attracted by the power and originality of Gelfand's approach to mathematics. He used the simplest grass-roots examples, but he could turn them around in a totally unexpected way.

Gelfand always paid special attention to students, who formed the majority of the seminar audience. From time to time he would repeat: "My seminar is for high school students, decent undergraduates, bright graduates, and outstanding professors." One of the best descriptions of the seminar was given by $\mathrm{S}$. Gindikin [3]. You may also see the notes by Zelevinsky and me in this collection (my notes will appear in

Gelfand, circa 1950 the next issue). Dusa McDuff described the seminar impressions of a young foreigner.

The seminar also served as a constant supply of Gelfand's collaborators, who were already familiar with Gelfand's style and his way of thinking. Their roles were quite different. Sometimes they would discuss specific examples, sometimes very vague ideas; sometimes they would bring their own suggestions that would be ridiculed, torn apart, turned upside down, and then transformed into something exquisite. Only a few could bear the task, but the pool of mathematicians in Moscow was enormous.

The seminar was a reflection of Gelfand's passion to teach, as he tried to teach everyone and everywhere. Among his former students are F. Berezin, J. Bernstein, E. Dynkin, A. Goncharov, D. Kazhdan, A. Kirillov, M. Kontsevich, and A. Zelevinsky. The number of his informal students is hard to estimate.

From the early period of his life also comes Gelfand's interest in education, especially in education for school students living far from research centers. He was among the founders of the Moscow Mathematical Olympiads and later organized his famous Mathematics School by Correspondence for middle and high school students (see the recollections by Sergei Tabachnikov in the second part of this article). Gelfand founded, ran, and wrote several textbooks for the school. His university textbooks Linear Algebra and Calculus of Variations (written with S. Fomin) also bear the imprint of his style and personality.

It is hard to describe all of Gelfand's achievements in mathematics. He left his unique and powerful imprint everywhere (excluding, probably, mathematical logic). Some (but far from all) of his breakthroughs are described here by Simon Gindikin, David Kazhdan, Bertram Kostant, Peter Lax, Isadore Singer, and Anatoly Vershik. But Gelfand's interests spread far beyond pure and applied mathematics. He left a number of papers in biology, physiology, medicine, and other fields.

Gelfand was the first to obtain the Wolf Prize, in 1978 (together with C. L. Siegel), and had many other awards, including the Kyoto Prize (1989) and the MacArthur Fellowship (1994). He was elected to all leading academies and had honorary degrees from many universities.

This collection of articles about Gelfand also contains an impression of the Gelfand seminar by a foreigner (Dusa McDuff), a look at the Gelfand school from inside (Andrei Zelevinsky and Vladimir Retakh), a description of Gelfand's School by Correspondence (Sergei Tabachnikov), and an essay, "Gelfand at 92" by Mark Saul.

\section{References}

[1] A talk with professor I. M. Gelfand, recorded by V. Retakh and A. Sosinsky, Kvant (1989), no. 1, 3-12 (in Russian). English translation in: Quantum (1991), no. 1, 20-26.

[2] A. SAKHARov, Memoirs, Alfred Knopf, New York, 1990.

[3] S. Gindikin, Foreword to I. M. Gelfand Seminar, Advances in Soviet Mathematics, vol. 16, Part 1, Amer. Math. Soc., Providence, RI, 1993.

\section{M. Singer}

\section{M. Gelfand}

Israel Gelfand was one of the most influential mathematicians of the twentieth century-I dare say, the most outstanding in the last sixty years.

Unfortunately, our society neither understands nor appreciates mathematics. Despite its many applications, despite its intellectual power which has changed the way we do science, mathematicians are undervalued and ignored. Its practitioners, its leaders go unrecognized. They have neither power nor influence. Watching the negative effects

I. M. Singer is Emeritus Institute Professor at the Massachusetts Institute of Technology. His email address is ims@math.mit.edu.

Adapted from: I. M. Singer, "Tribute to I. M. Gelfand", Progress in Mathematics, vol. 132, Birkhäuser Boston, 1995. 
popularity causes in other fields and looking at the few superficial articles about mathematics, I think it is just as well.

Faced constantly with problems we can't solve, most mathematicians tend to be modest about themselves and their accomplishments. Perhaps that is why we have failed to recognize a giant in our midst. I won't compare Gelfand with other outstanding mathematicians or scientists of the twentieth century; if I did, you would start checking for yourselves whether you agree with me. But focus on my point-we had a giant in our midst. I turn to other fields to find comparable achievements: Balanchine in dance or Thomas Mann in literature or Stravinsky, better still, Mozart in music, but for me, a better comparison is with artists like Cezanne and Matisse. I commend to you the great poet Paul Rilke's letter on Cezanne. He said, "Paul Cezanne has been my supreme example, because he has remained in the innermost center of his work for forty years... which explains something beyond the freshness and purity of his paintings" (of course, much longer for Gelfand).

Evoking Matisse is perhaps more apt. A Matisse is breathtaking. No matter what his personal circumstance, he turns to new frontiers with joy and energy. Particularly outstanding is his later work: Jazz and the remarkable "papier-decouples", efforts done in the early 1880 s.

Gelfand always dazzled us with new and profound ideas. One of his latest works, the book with Kapranov and Zelevinsky, is a major effort that maps out new directions for decades to come.

In preparing this article, I asked many people for topics I should emphasize. You will be interested in what happened. First, there was little intersection in the subjects my correspondents chose. Second, everyone gave me a five- to twenty-minute enthusiastic lecture on the essence of Gelfand's contribution-simple and profound.

Reviewing Gelfand's contributions to mathematics is an education. Let me remind you of some of his main work.

1. Normed rings

2. C*-Algebras (with Raikov) - the GNS Construction

3. Representations of complex and real semisimple groups (with Naimark and Graev)

4. Integral Geometry-Generalizations of the Radon Transform

5. Inverse scattering of Sturm-Liouville systems (with Levitan)

6. Gelfand-Dickey on Lax operators and KdV

7. The treatises on generalized functions

8. Elliptic equations

9. The cohomology of infinite dimensional Lie algebras (with Fuchs)
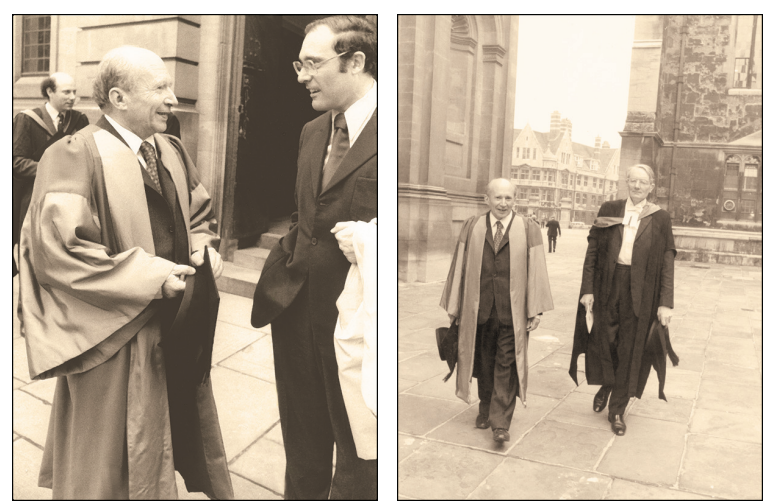

After receiving honorary degree at Oxford in 1973. Left with Gian-Carlo Rota.

10. Combinatorial characteristic classes (beginning with MacPherson)

11. Dilogarithms, discriminants, hypergeometric functions

12. The Gelfand seminar

It is impossible to review his enormous contributions in a short note. I will just comment on a few results that affected me.

As a graduate student, one of the first strong influences on me was Gelfand's normed ring paper. Marshall Stone had already taught us that points could be recaptured in Boolean algebras as maximal ideals. But Gelfand combined analysis with algebra in a simple and beautiful way. Using the maximal ideal in a complex commutative Banach algebra, he represented such algebras as algebras of functions. Thus began the theory of commutative Banach algebras. The spectral theorem and the Wiener's Tauberian theorem were elementary consequences. I was greatly influenced by the revolutionary view begun there.

A natural next step for Gelfand was the study of noncommutative $C^{*}$-algebras. He represented such algebras as operator algebras using the famous GNS construction. It seemed inevitable to find unitary representations of locally compact groups using their convolution algebras. The representation theory of complex and real semisimple Lie groups followed quickly. What struck me most was the geometric approach Gelfand and his coworkers took. Only recently it appears this subject has become geometric again.

In 1963 twenty American experts in PDE were on their way to Novosibirsk for the first visit of foreign scientists to the academic city there. It was in the midst of Khrushchev's thaw. When I learned about it, I asked whether I could be added to the list of visitors, citing the index theorem Atiyah and I had just proved. After reading his early papers, I wanted to meet Gelfand. Each day of my two-week stay in Novosibirsk I asked Gelfand's 


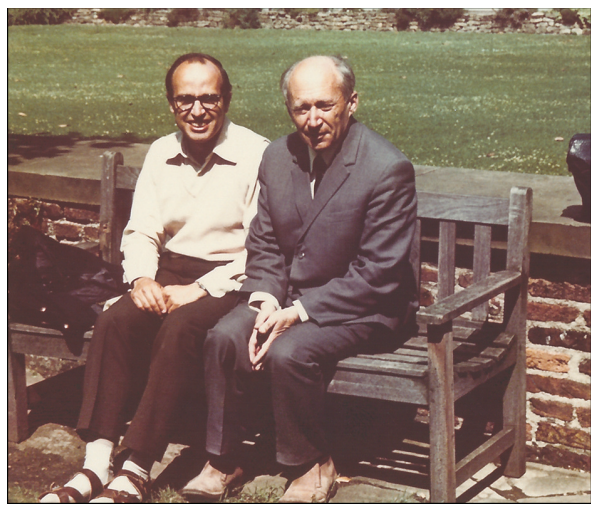

With M. Atiyah, 1973.

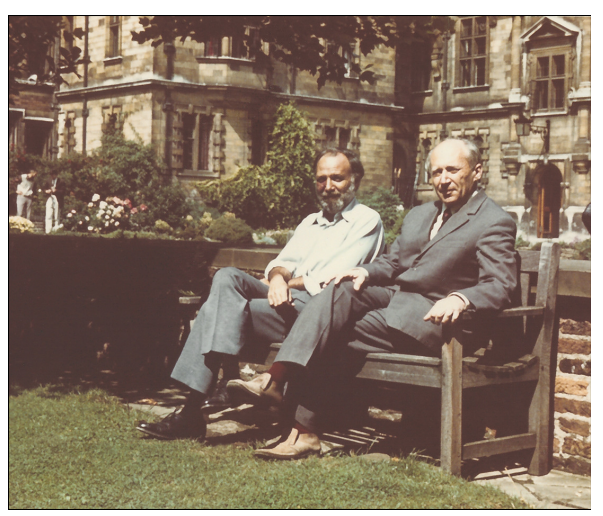

With I. M. Singer, 1973. students when he was coming. The response was always "tomorrow". Gelfand never came. I sadly returned to Moscow. When I got to my room at the famous Hotel Ukraine, the telephone rang and someone said Gelfand wanted to meet me, could I come downstairs. There was Gelfand. He invited Peter Lax and me for a walk. During this walk, Peter tried to tell Gelfand about his work on $S L(2, R)$ with Ralph Phillips. Gelfand tried to explain his own view of $S L(2, R)$ to Peter, but his English was inadequate. (He was rusty; within two days his English was fluent.) I interrupted and explained Gelfand's program to Peter. At the corner Gelfand stopped, turned to me and said, "But you are my student." I replied, "Indeed, I am your student." (By the way, Gelfand told me he didn't come to Novosibirsk, because he hated long conferences.)

Although it was an honor to be Gelfand's student, it was also a burden. We tried to imitate the depth and unity Gelfand brought to mathematics. He made us think harder than we believed possible. Gelfand and I became close friends in a matter of minutes, and we remained so ever since. I was ill in Moscow, and Gelfand took care of me.

I didn't see him again for ten years. He was scheduled to receive an honorary degree at Oxford, where I was visiting. It was unclear that he would be allowed to leave the Soviet Union to visit the West. I decided not to wait and returned home. A week later, I received a telegram from Atiyah: Gelfand was coming - the queen had asked the Russian ambassador to intercede. I flew back to England and accompanied Gelfand during his visit-a glorious time. Many things stand out, but I'll mention only one: our visit to a Parker fountain pen store. Those of you who have ever shopped with Gelfand will smile; it was always an unforgettable experience. Within fifteen minutes, he had every salesperson scrambling for different pens. Within an hour I knew more about the construction of fountain pens than I ever cared to know and had ever believed possible! Gelfand's infinite curiosity and focused energy on details were unbelievable; those, coupled with his profound intuition of essential features, are rare among human beings. He was beyond category.

Talking about Oxford, let me emphasize Gelfand's paper on elliptic equations. In 1962 Atiyah and I had found the Dirac operator on spin manifolds and already had the index formula for geometric operators coupled to any vector bundle, although it took another nine months to prove our theorem. Gelfand's paper was brought to our attention by Smale. It enlarged our view considerably, as Gelfand always did, and we quickly realized, using essentially the Bott periodicity theorem, that we could prove the index theorem for any elliptic operator.

I should also mention the application of Gelfand's work to physics: Gelfand-Fuchs, for example, on vector fields on the circle, the socalled Virasoro algebra, which Virasoro did not in fact define. Although I mentioned Gelfand-Dickey, I have to stress its influence on matrix model theory. And I have to describe how encouraging he was and how far ahead of his time he was in understanding the implication of a paper which seemed obscure at the time.

Claude Itzykson told me that his famous paper with Brezin, Parisi, and Zuber that led to triangulating moduli space at the beginning went unnoticed by scientists. The authors then received one request for a reprint-from Gelfand.

Ray and I were very excited about our definition of determinants for Laplacian-like operators and its use in obtaining manifold invariants-analytic torsion. The early response in the United States was silence; Gelfand sent us a congratulatory telegram.

In conclusion, I want to mention one special quality of Gelfand. He was a magician. It is not very difficult-not very difficult at all-for any of us mere mortals to keep the difference in our ages a constant function of time. But with Gelfand, when I met him at his fiftieth, and in his sixtieth, I thought he was older than I. Ten years later, I felt that we were the same age. Later it became clear to me that Gelfand was, in fact, much younger than most mathematicians. 


\section{David Kazhdan}

\section{Works of I. Gelfand on the Theory of Representations}

The theory of group representations was the center of interest of I. Gelfand. I think this is related to the nature of this domain, which combines analysis, algebra, and topology in a very intricate fashion. But this richness of the representation theory should not be taken as self-evident. To a great extent we owe this understanding to works of I. Gelfand, to his unique way of seeing mathematics as a unity of different points of view.

In the late thirties, when Gelfand started his mathematical career, the theory of representations of compact groups and the general principles of harmonic analysis on compact groups were well understood due to works of Hermann Weyl. Harmonic analysis on locally compact abelian groups was developed by Lev Pontryagin. The general structure of operator algebras was clarified by Murray and von Neumann. But the representation theory of noncompact noncommutative groups was almost nonexistent. The only result I know of is the work of Eugene Wigner on representations of the inhomogeneous Lorentz group. Wigner has shown that the study of physically interesting irreducible representations of this group can be reduced to the study of irreducible representations of its compact subgroups.

It was not at all clear whether the theory of representations of real semisimple noncompact groups is "good", i.e., whether the set of irreducible representations could be parameterized by points of a "reasonable" set, and whether the unitary representations can be uniquely decomposed into irreducible ones. The conventional wisdom was to expect that the beautiful theory of Murray-von Neumann factors is necessary for the description of representations of real semisimple noncompact groups. On the other hand, Gelfand, for whom Gauss and Riemann were the heroes, expected that the theory of representations of such groups should possess classical beauty.

Gelfand's first result in 1942 with D. Raikov in the theory of representations of groups is a proof of the existence of "sufficiently many" unitary representations for any locally compact group $G$. In other words, any unitary irreducible representation of $G$ is a direct integral of irreducible ones. The proof of this result is based on the very important observation that the representation theory of the group $G$ is identical to the theory of representations of the convolution algebra of

David Kazhdan is professor of mathematics at the Einstein Institute of Mathematics. His email address is kazhdan@math.huji.ac.i1. measures on $G$ with compact support and on an application of Gelfand's theory of normed rings.

Next, in the late forties, there was a stream of papers (most of them jointly with M. Naimark) that developed the main concepts of the rep-

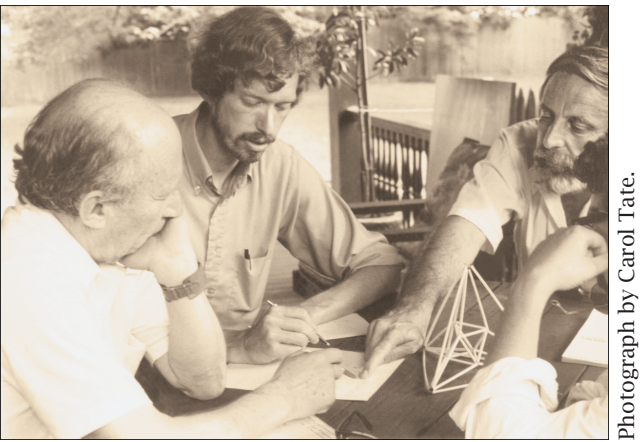

On Raoul Bott's porch, 1976. Left to right, I. Gelfand, R. MacPherson, R. Bott, D. Kazhdan. resentation theory of noncompact classical groups $G$. It would be a much simpler task to describe the concepts that appeared later than to describe the richness of this work.

Gelfand believed that the space $\hat{G}$ of irreducible representations of $G$ is a reasonable "classical" space. If I understand correctly, the first indication of the correctness of this intuition came from the theory of spherical functions, developed in the early forties but published only in 1950 . Let $K \subset G$ be the maximal compact subgroup and $\hat{G}_{0} \subset \hat{G}$ be the subset of irreducible representations of class 1 (that is, the representations $(\pi, V)$ of $G$ such that $V^{K} \neq 0$ ). Gelfand observed that the subset $\hat{G}_{0}$ is equal to the set of irreducible representations of the subalgebra of two-sided $K$-invariant functions on $G$, proved the commutativity of this algebra, and identified the space of its maximal ideals with the quotient ${ }^{L} T / W$ where ${ }^{L} T$ is the torus dual to the maximal split torus $T \subset G$ and $W$ is the Weyl group. The generalization of this approach developed in the fifties by Harish-Chandra and Godement led to the proof of the uniqueness of the decomposition of any representation of the group $G$ into irreducibles.

Given such a nice classification of irreducible representations of class 1 , it was natural to guess that the total space $\hat{G}$ is also an algebraic variety. But for this purpose one had to find a way to construct irreducible representations of $G$. Gelfand introduced the notion of parabolic induction (for classical groups) and, in particular, studied representations $\pi_{\xi}$ of $G$ induced from a character $\xi$ of a Borel subgroup $B \subset G$. He showed that, for generic character $\xi$ of $T=B / U$, the representation $\pi \xi$ is irreducible and that the representations $\pi \xi, \pi \xi^{\prime}$ are equivalent if and only if the characters $\xi$, $\xi^{\prime}$ of $T$ are conjugate under the action of the Weyl group $W$. The proof is based on the decomposition $G=\bigcup_{w \in W} B w B$ for classical groups. This decomposition was extended by Harish-Chandra to the case of an arbitrary 


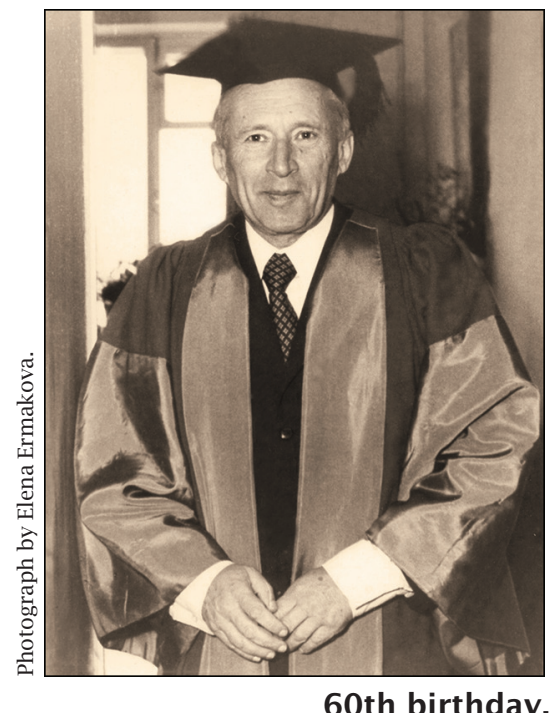

semisimple group and is known now as the Bruhat decomposition.

This construction gives many irreducible representations. But how to show that not much is missing? In the case of a compact group $G$ it is well known that all the representations of $G$ are constituents of the regular representation. Therefore, to see that a list of representations $\pi_{a}, a \in A$, of $G$ is complete, it is sufficient to show that one can write the delta function $\delta_{e}$ on $G$ as a linear combination of the characters $\operatorname{tr}\left(\pi_{a}\right)$. But for representations $(\pi, V)$ of a noncompact group $G$, which are typically infinite-dimensional, the trace of the operator $\pi(g), g \in G$, is not defined. The ingenious idea of Gelfand was to define characters $\operatorname{tr}(\pi)$ as distributions. That is, he showed that for any smooth function $f(g)$ with compact support, the operator

$$
\pi \xi(f):=\int f(g) \pi \xi(g) d g
$$

is of trace class and defined the distribution $\operatorname{tr}(\pi)$ by $\operatorname{tr}(\pi)(f):=\operatorname{tr}(\pi(f))$. Now one could look for a $W$-invariant measure (called the Plancherel measure) $\mu_{X}$ on the space $X$ of unitary characters of $T$ such that

$$
\delta_{e}=\int \xi \in X \operatorname{tr}(\pi \xi) \mu_{X}
$$

It is not difficult to see that such a measure $\mu_{X}$ is unique (if it exists), and the knowledge of $\mu_{X}$ is equivalent to the explicit decomposition of the regular representation $L^{2}(G)$ into irreducible ones.

In a series of joint works with M. Naimark, Gelfand was able to guess a beautiful algebraic expression for the Plancherel measure $\mu_{X}$ in the case of classical complex groups and to prove equality 1 by very intricate explicit calculations-a great reward for difficult work.

As a continuation of this series of works, Gelfand posed a number of questions (he was able to answer them only in particular cases) which influenced the development of representation theory for many years.

1. In joint work with M. Graev, Gelfand classified generic irreducible representations of the group $S L(n, \mathbb{R})$. They found that $\hat{G}$ is a union of pieces called series which correspond to conjugacy classes of maximal tori. Moreover, the series corresponding to nonsplit tori have realizations in spaces of (partially) analytic functions. Gelfand conjectured that the analogous description of the space $\hat{G}$ should be true for all real semisimple groups and that it should be possible to realize discrete series in appropriate spaces of analytic functions. The first part of the conjecture was justified by Harish-Chandra, who constructed discrete series of representations for real semisimple groups and found the Plancherel measure concentrated on unitary representations induced from discrete series of Levi subgroups. The second part was modified by Langlands, who suggested the realization of discrete series (which exist if and only if there is a maximal compact torus $T^{c} \subset G$ ) in the space of cohomologies $H^{i}\left(G / T^{c}, \mathcal{F}\right)$ of homogeneous holomorphic vector bundles $\mathcal{F}$ on $G / T^{c}$ (while Gelfand considered only the realization in sections of such bundles).

2. In joint work with Graev, Gelfand constructed the analog of the Paley-Wiener theorem for groups $S L_{2}(\mathbb{C})$ and $S L_{2}(\mathbb{R})$ (that is, a decomposition of the representation of $G$ on the space $\mathbb{C}_{c}^{\infty}(G)$ of smooth functions with compact support) and raised the question about the extension of this result to other groups. This generalization was obtained by J. Arthur in 1983.

3. Also in joint work with Graev, Gelfand constructed the decomposition of representations of the group $S L_{2}(\mathbb{C})$ on the space $L^{2}\left(S L_{2}(\mathbb{C}) / S L_{2}(\mathbb{R})\right.$ and asked the question about the decomposition of representations of the group $G$ on $L^{2}(G / H)$ where $H \subset G$ is the set of fixed points of an involution. An extension of this result to arbitrary such pairs $(G, H)$ was achieved only recently (see the talk by P. Delorme at the ICM Congress, 2002).

4. Gelfand showed that many special functions, such as Bessel and Whittaker functions and Jacobi and Legendre polynomials, appear as matrix coefficients of irreducible representations. This interpretation of special functions immediately explains the functional and differential equations for these functions. It is clear now that (almost) all special functions studied in the nineteenth and twentieth centuries can be interpreted as matrix coefficients or traces of representations of groups or their quantum analogs (see, for example, papers of Tsuchiya-Kanie, Koelink, Noumi, Rosengren, Stokman, Sugitani, and others on representation theoretic interpretation of Askey-Wilson, Macdonald, and Koornwinder polynomials).

The next series of Gelfand's work (with M. Tsetlin) was on irreducible finite-dimensional representations of classical groups $G$. The classification of such representations $\left(\pi_{\lambda}, V_{\lambda}\right)$ was known, but Gelfand asked a new question, partially influenced by his interest in physics: how to find a "good" realization of these representations. In other words, how to find a basis in $V_{\lambda}$ which allows one to 
compute matrix coefficients of $\pi_{\lambda}(g), g \in G$, in these bases. Such a basis (Gelfand-Tsetlin basis) was constructed for irreducible representations of groups $S L_{n}$ and $S O_{n}$ and became the core of many works in representation theory and combinatorics.

Gelfand and Graev found the expression for the matrix coefficients of representations $\pi_{\lambda}$ in terms of discrete versions of $\Gamma$-functions. This realization of finite-dimensional representations has an important analog for infinite-dimensional representations of groups over local fields.

As part of the theory of finite-dimensional representations, Gelfand studied the ClebschGordan coefficients, which give a decomposition of tensor products of irreducible representations into irreducible components. He noticed (at least for $G=S L_{2}$ ) that Clebsch-Gordan coefficients of $G$ are discrete analogs of Jacobi polynomials which are matrix coefficients of irreducible representations of $G$. Possibly an explanation of this can be given by using the theory of quantum groups, where multiplication and comultiplication are almost symmetric to each other.

The next series of Gelfand's work was on representations of groups over finite and local fields $F$. The basic results here are the proof of the uniqueness of a Whittaker vector, the existence of a Whittaker vector for cuspidal representations of $G L_{n}(F)$, the construction of an analog of the Gelfand-Tsetlin basis for such representations, and the description of cuspidal representations of $G L_{n}(F)$ in terms of $\Gamma$-functions (joint work with M. Graev and D. Kazhdan). But I think that his most important work in this area is the complete description of irreducible representations of groups $S L_{2}(F)$ and $G L_{2}(F)$ for local fields with the residue characteristic different from 2 (joint work with M. Graev and A. Kirillov). They showed that irreducible representations of $G L_{2}(F)$ are essentially parameterized by conjugacy classes of pairs $(T, \xi)$ where $T \subset G L_{2}(F)$ is a maximal torus and $\xi: T \rightarrow \mathbb{C}$ is a character. Moreover, they found a formula for the characters $\operatorname{tr}_{T_{\xi}}(g)$ of these representations and an explicit expression for the Plancherel measure. A striking and until now unexplained feature of these formulas is that they are essentially algebraic. For example, the Mellin transform $L(g, t)$ in $\xi$ of $t r_{T_{\xi}}(g)$, which is a function of $G L_{2}(F) \times T$, is given by

$$
\begin{aligned}
L(g, t)= & \delta(\operatorname{det}(g), \mathbf{N} m(t)) \epsilon_{T} \\
& \times(\operatorname{tr}(g)-\mathbf{t} r(t)) /|\operatorname{tr}(g)-\mathbf{t} r(t)| .
\end{aligned}
$$

Here $T=E^{*}$ where $E$ is a quadratic extension of $F, \epsilon_{T}: F^{*} \rightarrow \pm$ is the quadratic character corresponding to $E$, $t r$ is the matrix trace, and $t r$ and $\mathrm{N} m$ are the trace and norm maps from $E$ to $F$.

The understanding of the existence of an intrinsic connection between the structure of irreducible representations of groups over local fields and number theory was greatly clarified by Langlands. On the other hand, a generalization of algebraic formulas for the Mellin transform of characters and for the Plancherel measure was never found.

In other work, Gelfand and Graev Talking at 90th birthday conference, found a description 2003.

of the irreducible representations of the multiplicative group $D^{*}$ of quaternions over $F$ as induced from 1-dimensional representations of appropriate subgroups. This was of constructions of irreducible representations of $D^{*}$ that were later generalized by $\mathrm{R}$. Howe to other $p$-adic groups.

The description of representations of the groups $S L_{2}(F)$ and $G L_{2}(F)$ for local fields is presented in the book Generalized Functions, Volume 6 written with Graev and I. Piatetski-Shapiro. In the same book, Gelfand developed the theory of representations of semisimple adelic groups $G\left(\mathbb{A}_{K}\right)$ for global fields $K$. He defined the cuspidal part

$$
L_{0}^{2}\left(G\left(\mathbb{A}_{K}\right) / G(K)\right) \subset L^{2}\left(G\left(\mathbb{A}_{K}\right) / G(K)\right)
$$

of the space of automorphic forms, proved that the representation of $G\left(\mathbb{A}_{K}\right)$ on $L_{0}^{2}\left(G\left(\mathbb{A}_{K}\right) / G(K)\right)$ is a direct sum of irreducible representations, and developed a representation-theoretical interpretation of the theory of modular forms. The work of Langlands is very much influenced by these results of Gelfand.

It became clear that generic representations of any semisimple Lie group or Lie algebra almost do not depend on a choice of a particular group, so Gelfand tried to find a way to express this similarity in an intrinsic way. In a series of papers with A. Kirillov he studied the structure of the skew-field $F(\mathfrak{g})$ of fractions for the universal enveloping algebra of a Lie algebra $\mathfrak{g}$. He found that the skew-fields $F(\mathfrak{G})$ are almost defined by the transcendence degree of the center $Z(\mathfrak{g})$ (equal to the rank $r(\mathfrak{g})$ of $\mathfrak{g})$ and by their Gelfand-Kirillov dimension (equal to $(\operatorname{dim}(\mathfrak{g})-r(\mathfrak{g})) / 2)$. These results are the foundation of works of A. Joseph on the structure of the category of Harish-Chandra modules.

The last series of work of Gelfand on representation theory was on category $\mathcal{O}$ of representations of a semisimple Lie algebra. This category of representations was defined by Verma, but the 
basic results are due to J. Bernstein, I. Gelfand, and S. Gelfand. They constructed a resolution of finite-dimensional representations by Verma modules $V_{w}, w \in W$ (known as the BGG-resolution), discovered the duality between irreducible and projective modules in the category $\mathcal{O}$, and found the relation between the category $\mathcal{O}$ and the category of Harish-Chandra modules. These results form the cornerstone of the theory of representations of semisimple Lie algebras and their affine analogs.

However, their main discovery was the existence of a strong connection between algebraic geometry of the flag space $\mathcal{B}$ of a semisimple group and the structure of the category $\mathcal{O}$. For example, they showed that there is an embedding of $V_{w}$ into $V_{w^{\prime}}$ if and only if the Bruhat cell $B w B \subset \mathcal{B}$ belongs to the closure of $B w^{\prime} B$. This connection between algebraic geometry and the category of representations is the basis for the recent geometric theory of representations.

There are other important works of Gelfand on representation theory (such as indecomposable representations of semisimple Lie groups, models of representations, and representations of infinite-dimensional groups), but I want to mention two series of works that originated in representation theory but have an independent life. The appearance of such works is very natural, since for Gelfand representation theory was a part of a much broader structure of analysis.

Integral geometry is an offshoot of representation theory. The proof of the Plancherel theorem for complex groups is equivalent to the construction of the inversion formula, which gives the value of a function in terms of its integrals over horocycles. Gelfand (in a series of joint works with Graev, Z. Shapiro, S. Gindikin, and others) found inversion formulas for reconstruction of the value of a function on a manifold in terms of its integrals over an appropriate family of submanifolds. The existence of such inversion formulas found applications in such areas as symplectic geometry, multi-dimensional complex analysis, algebraic analysis, nonlinear differential equations, and Riemannian geometry, as well as in applied mathematics (tomography). (A more detailed description of Gelfand's work on integral geometry is given by S. Gindikin in the current issue of the Notices.)

Analogously, the work of Gelfand with V. Ponomarev and, later, with J. Bernstein on quivers was motivated by the problems in representation theory-the description of indecomposable representations for the Lorentz group. But the inner development of this subject led to a beautiful and deep theory which later made a full circle in works of Ringel, Lusztig, and Nakajima and became the foundation for geometric representation theory of Lie algebras and quantum groups.

\section{Anatoly Vershik}

\section{Gelfand, My Inspiration}

The achievement of Israel Moiseevich Gelfand was an unusual phenomenon of twentieth-century mathematics. His name must be included on any short list of those who formed the mathematics of that century. He pioneered many new ideas and created whole new provinces of knowledge. An exceptional intuition, a depth and breadth of thought, a liveliness in comprehending mathematics-these were his chief qualities.

But the most important quality of this wonderful mathematician was his ability to inspire others. Many other mathematicians, both in Russia and abroad, felt an amazing sense of inspiration in talking to or corresponding with Gelfand. His ideas and his advice stimulated many discoveries and much research done by others.

\section{Gelfand and Leningrad Mathematics}

I was a student in Leningrad in the 1950s, when Gelfand's name was well known to all Leningrad mathematicians, including students interested in the subject. This is not surprising. The interests of most Leningrad mathematicians (including L. V. Kantorovich, V. I. Smirnov, G. M. Fikhtengoltz, and others) centered on functional analysis and its applications, and this "Leningrad approach" was one of the branches of functional analysis that developed in the Soviet Union in the years 1930-1950. I. M. Gelfand was an iconic figure in this development.

The Leningrad approach differed from that taken in Moscow (by A. N. Kolmogorov, I. M. Gelfand, L. A. Lyusternik and A. I. Plessner) and in the Ukraine (by M. G. Krein and N. I. Akhiezer). It was centered around the theory of functions, operator theory, and its applications to differential equations and methods of computation. The main difference between the Moscow approach and the other two was the study advocated by Gelfand of infinite-dimensional representations of groups and algebras and an interest not so much in Banach spaces as in general problems of noncommutative Fourier analysis and, more broadly, in a synthesis of algebra, classical analysis, and the theory of functions. These ideas were not present in Leningrad for many years.

Anatoly Vershik is professor of mathematics at the St. Petersburg Department of the Steklov Mathematical Institute RAS. His email address is vershik@pdmi.ras.ru.

This segment of the article was translated from the Russian by Vladimir Retakh and Mark Saul. 
Gelfand's fame and authority went unchallenged at Leningrad seminars. While still young, Gelfand had broken into the elite club of Moscow mathematicians of the highest level, amazing everyone with his theory of maximal ideals of commutative Banach algebras (the so-called "Gelfand transform"), and he remained a leader of this club until his death.

Among Leningrad mathematicians Gelfand especially valued L. V. Kantorovich, D. K. Faddeev, and V. A. Rokhlin, whom he remembered from his student days. Like Kantorovich, Gelfand played an active role in the atomic research project and generally knew the work of Kantorovich on functional analysis. Both these outstanding mathematicians did much work on applied problems.

In 1986, at the funeral of Kantorovich, Gelfand told me that of all of Kantorovich's results, the one that impressed him the most was his pioneering work on linear programming. That was my view as well. On the other hand, when I asked Kantorovich to submit my paper to Doklady in 1971, he noted that the paper really represented the work of Gelfand and that it was disgraceful that Gelfand was not still a full member of the Soviet Academy of Science. Gelfand had direct contact with only a few of the younger Leningrad mathematicians in addition to me-in particular, with L. D. Faddeev and M. S. Birman.

\section{My First Acquaintance with Gelfand's Work}

The first significant mathematical papers that I studied were the papers of I. M. Gelfand, D. A. Raikov, and G. E. Shilov (Gimdargesh, as I called them) on commutative normed rings and their papers that followed on generalized Fourier analysis. This theory was a mathematical awakening for me. I was enchanted by its beauty and simplicity, its generality and depth.

Before this I had been hesitant. I could join the Department of Algebra, where I attended lectures by D. K. Faddeev. Or I could work in the Department of Analysis, where my first mentor was G. P. Akilov and where I might choose to specialize in complex analysis (V. I. Smirnov, N. A. Lebedev) or real and functional analysis (G. M. Fikhtengoltz, L. V. Kantorovich, G. P. Akilov). Now functional analysis was my only choice. And my interest was mostly in the analysis of the Moscow schoolGelfand's functional analysis, as I described earlier. Since that time the work of Gelfand and his school in various areas have become my mathematical guide.

One could say a lot about the great variety of Gelfand's interests and works. In fact, he was interested in everything: biology, music, and politics, not just mathematics. I would like to write here about the area of his mathematical activity that was closest to my interests and about the problems on which I was fortunate to work with him.

I would go so far as to say that the main legacy of the work of Gelfand's first

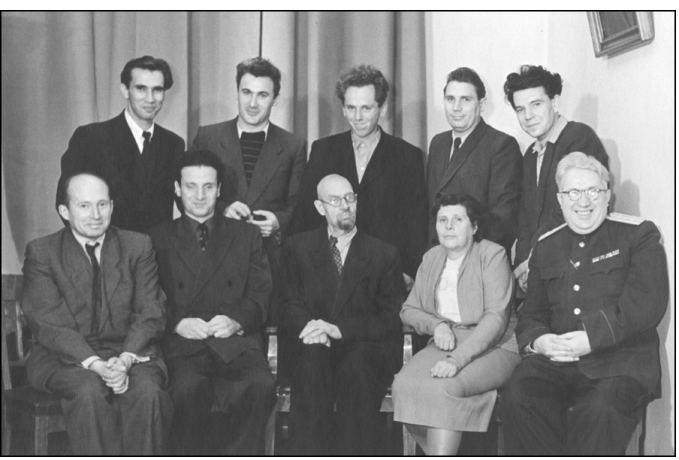

Lab of Function Theory at Moscow State University, circa 1958. Left to right sitting, I. Gelfand, Polyakov, D. E. Menshov, N. K. Bari, G. P. Tolstov; standing, P. L. Ulyanov, A. G. Kostychenko, F. A. Berezin, G. E. Shilov, R. A. Minlos. period was his foundation (along with the group of students he led) of the theory of normed commutative rings (commutative Banach algebras, as they are now called) and, most importantly, the theory of unitary infinite-dimensional representations of locally compact groups. These papers became classics of mathematics despite the youth of the authors.

As a byproduct of this theory, Gelfand and Naimark gave a definition of general $C^{*}$-algebras that is now in common usage. Once, as early as the 1970s, Gelfand said to me, "If I could only talk to von Neumann, I would explain to him why our $C^{*}$-algebras are more important than $W^{*}$-algebras (von Neumann algebras)." I can add that Gelfand was always more interested in topological and smooth problems (which are connected with $C^{*}$-algebras) than in theoretical metric problems (which are formulated in terms of $W^{*}$-algebras). The representation theory that will forever be connected with his name always was a favorite subject of Gelfand's, but his ideas about relationships and details within this theory kept changing. Among his wonderful traits was a fearless irony towards his own earlier results. When I told him that as a student I was impressed by his work on Banach algebras, Gelfand replied, "But why did we consider only maximal and not prime ideals?" This remark is similar to one he made in a talk in the 1970s, when I mentioned to him my interest in his early papers. "I am always praised for my work that was done five or more years ago, and the same people are not satisfied with what I am doing right now," he explained about his not uncommon critics. In fact, he was always ahead of his time, he was always creating a new fashion.

I remember being strongly influenced by Gelfand's talk in 1956 about problems in functional analysis (he was probably following the 
example of a similar talk by von Neumann at the International Congress of Mathematicians in 1954). In this address he posed several general problems and talked about their relationship to various mathematical phenomena. One of these problems was a conjecture about a possible connection between Wiener measures and von Neumann factors and of its possible utility in future work. "Such beauty must not perish unused," noted Gelfand. I was a student at that time and I was deeply impressed by this strong assertion of the primacy of aesthetic estimation of mathematical theories above other criteria.

\section{A Few Comments on Gelfand's Work of the 1950s and 1960s}

Perhaps Gelfand's highest achievement was the theory of infinite-dimensional unitary representations of complex semisimple Lie groups, created together with M. A. Naimark, M. I. Graev, and others.

It is hardly possible to comment on this development completely here, but it is worth noting that Gelfand faced many difficulties common to all pioneers: many details had to be clarified later or proofs rewritten, and so it would happen that attributions of priority or authorship were given to other researchers. But one must remember that Gelfand was the first to initiate and develop this huge area of mathematics, which is so useful in physics. There was no theory of infinite-dimensional representations before Gelfand's. From reading the recently published diaries of A. N. Kolmogorov, one can understand how impressed the world of mathematics was by these works in the 1940s. It is remarkable how confident Gelfand was in starting up completely new areas.

Gelfand's next great passion in the late 1950s and 1960s - an interest common to many centers of mathematics-was the theory of distributions of L. Schwartz (called in Russia "generalized functions"). Several Russian mathematicians had a keen interest in the subject but with a touch of bitterness and perhaps chagrin. The reason was simple: Gelfand, and before him N. M. Gyunter (a famous Petersburg mathematician) and L. V. Kantorovich and certainly S. L. Sobolev in fact not just made use of but created the theory of generalized functions. In one of his lectures in Leningrad in the early1960s, Gelfand stated directly that he and Naimark used distributions in their work on representation theory. However, the merit of L. Schwartz's work is that he understood the importance of the general theory and substantiated it with many examples. It is important to add that, as often happens in the theory of functions, this reformulation actually gave a new language and new understanding to many concepts.
However, the hope that arose at that time that this reformulation would give essentially new results did not exactly come true, and we cannot talk about a serious change from the theory of Banach spaces to the theory of locally convex spaces, despite the expectations of that time.

The celebrated six-volume series of books Generalized Functions by Gelfand and his coauthors demonstrated their unparalleled depth of understanding of the subject. The breadth of the area covered, from partial differential equations to representations and number theory, is characteristic of Gelfand's style.

In particular, Gelfand was among the first to understand the importance of turning from Banach's methods in functional analysis to more general linear topological (nuclear) spaces and their value for the spectral theory of operators (the GelfandKostyuchenko theorem) and the theory of measure in linear spaces (the Minlos theorem). Gelfand also immediately understood the usefulness of generalized stochastic processes (Gelfand-Ito processes). This stimulated the construction of the theory of measures in linear spaces started by A. N. Kolmogorov in the 1930s. The famous "holy trinity" (triples of Hilbert spaces), generalized spectral decompositions, an original approach to Levi processes and to Gelfand-Segal constructions, quasi-invariant measures, and so on were studied and developed by hundreds of mathematicians.

\section{My First Meeting with Gelfand}

Not counting a few brief discussions with Gelfand during the Mathematical Congress in 1966 or in the late 1960s or on my rare visits to his seminar when I happened to be in Moscow, our first close meeting took place in the spring of 1972 when I came to Moscow for two months. After the seminar I walked to his home with several participants. I started talking about the work I had recently started on asymptotic statistics of the lengths of cycles of random permutations. This paper initiated a long series written by me and my students on what I later called the asymptotic theory of group representations. Gelfand was interested and invited me to his home the next day to continue the conversation. I remember that Dima Kazhdan, who was also there, understood me sooner than Gelfand, who often asked me to repeat what I had said. His active and sometimes aggressive questioning was very helpful to the development of the theme and the improvement of the talk, if you could ignore the form of the criticism. But I had long ago heard about Gelfand's manner in such things. In talking about mathematics he would openly express his displeasure to his listener. One of his expressions, which is useful in the upbringing of young mathematicians, was "Keep your work and 
your self-esteem separate." In other words, do not take even harsh intellectual criticism personally.

I told Gelfand about my plans to study the symmetric group and its representations. My motivation for this research lay not only in the study of the subject by itself but also in its applications to optimization theory, linear programming, and combinatorics. (At that time I worked in the Department of Computational Mathematics and Operational Research.) There was also a way of connecting asymptotic theory with ergodic theory. In our discussions, Gelfand remarked that everything seemed to be clear with regard to representations of finite groups, then started talking enthusiastically about symmetric functions. He recommended that I look at a mysterious paper of E. Thoma about characteristics of the infinite symmetric group, which were of special interest to me.

Many things about the symmetric group were unclear to me, and I was not satisfied with the classical exposition of the theory of its representations despite the fact that it had been developed by Frobenius, Schur, and Young, and also by such giants as von Neumann and H. Weyl. It seemed to me then that the foundations of this theory and its relations with combinatorics were not yet clear. Gelfand gave his final conclusion many years later, after he had gotten to know and appreciate my papers with S. Kerov and later with A. Okounkov. He said something like, "It is all clear now." But that was all years later. In fact, the main idea of my approach was, roughly speaking, an application of the Gelfand-Tsetlin method, created in the early 1950s for representation of compact Lie groups, to the theory of symmetric groups. It is worth noting that a dissatisfaction with established theory often begins with those studying the theory in their mature years. That is what happened in my case. I started to study representations theory later than usual and got a certain advantage from this.

In Moscow, during our first conversation at his home, Gelfand once again surprised me. He repeated part of our discussion to Dima Kazhdan, who had come in later, and said, as if it had already been done, that I was studying the asymptotics of Young diagrams. However, I had not mentioned this and was just planning to do it. When I corrected him, Gelfand said, "Yeah, yeah, but you will do it later." This unique ability of Gelfand to see "three meters under the ground where his interlocutor was standing" was one of his most remarkable qualities. It instilled trust in his judgment by many who got to know him, but also drove off many who feared his ability to see through them. He immediately and (usually) correctly guessed what his companions were about to say or what they had in mind but did not express, and generally knew how the discussion would turn out. There are many examples of this, the most striking was his mathematical intuition, which allowed him to guess the result (often without calculations) and to foresee what to expect

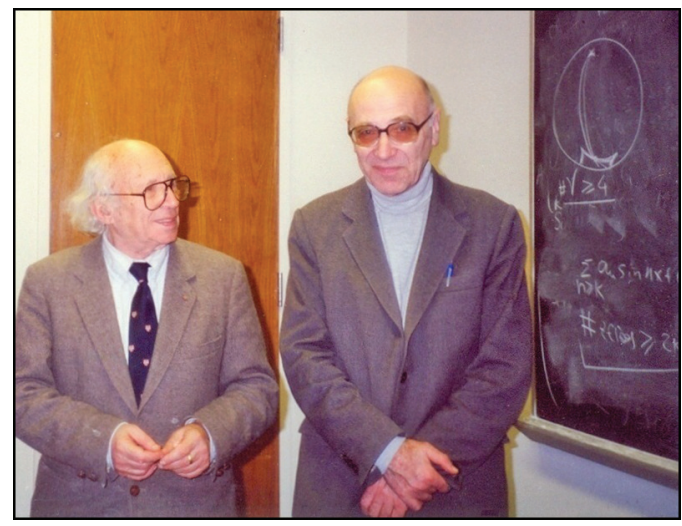

With M. I. Graev, 1999. from a given method or even from the further work of a mathematician or a whole team. During that discussion Gelfand approved of my ideas (later called asymptotic representations theory). Many of these plans were then acted on by S. Kerov, G. Olshanski, A. Okounkov, A. Borodin, and a number of Western mathematicians.

At Gelfand's seminar in 1977 I talked with S. Kerov about my results on the limiting form of Young diagrams and about our approximation approach to representations of the infinite symmetric group. At that time Gelfand, Graev, and I had already started our work on the representations of current groups. I. M. always commented on every talk at his seminar. During my talk he said (and repeated this many times) that combinatorics is becoming a central part of mathematics of the future. As a permanent seminar participant said to me, "He was excited."

\section{Our Collaboration}

Let me return to 1972 and to the history of our collaboration. After our conversation I said goodbye and intended to leave for Leningrad. In Moscow I always stayed with my old friend, the virologist N. V. Kaverin. He once attended a Gelfand seminar in biology. Gelfand remembered him, but they had no other contact. I mentioned to Gelfand that I was staying with Kaverin. Suddenly, on the day of my departure, Gelfand called me at Kaverin's (having found the phone number with some difficulty ${ }^{1}$ ) and asked me to come over immediately. He also invited M. I. Graev, and during our long walk together started to talk about a construction of noncommutative integrals of representations of semisimple groups, primarily for $S L(2, R)$. He said that he had been incubating this problem for a long time and had suggested it to his other students, but he was confident that the problem was perfect for me.

\footnotetext{
${ }^{1}$ There was no "official" way to get someone's telephone number in Moscow at the time.
} 


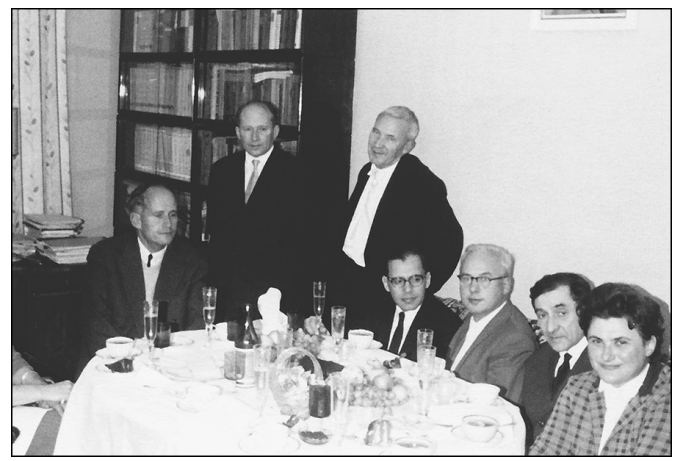

At the time of the ICM, 1966. Second from left, I. Gelfand; third from left, A. Kolmogorov; fifth from left, S. V. Fomin; far right, $\mathbf{O}$. A. Oleinik.
I was a bit surprised that Gelfand was aware of my knowledge of the representation theory of Lie groups, in particular of $S L(2, R)$. We had not talked about this. But Gelfand was right: the problem was posed at just the right time for me. In the early 1970s, independent of my other work, I was lecturing on representations of groups, $C^{*}$-algebras, and factors. Perhaps Gelfand had heard about this, but not from me. More likely, this was the result of his ability to foresee things that I mentioned earlier. Gelfand said that in order to construct the multiplicative integral of representations we have to study "infinitesimal" representations, i.e., a neighborhood of the identity representation. In the summer of 1972 Gelfand came to Leningrad for the thesis defense of M. Gromov, and I told him about my preliminary experiments with the Heisenberg group, where a construction of the integral by other methods was already known. In December of 1972 we found a solution, a spherical function of the required representation of $S L(2, R)$, or the "canonical state", as Gelfand suggested we call it. I came to Moscow and we drafted the text. The problem was solved in a few months. The next spring Gelfand came to Leningrad with M. I. Graev and stayed with us. My domestic helpers prepared food as instructed by Zorya Yakovlevna. ${ }^{2}$ While we were working on the paper with Graev, I. M. talked with my wife, Rita, about his favorite music and paintings. Of course, we went to the Hermitage Museum, where Gelfand talked a lot about a painting of El Greco (Saints Peter and Paul) and where we also discussed how to continue our work.

I recall next Gelfand's last visit to Leningrad in 1984. I invited him to talk about his own life at a meeting of the Leningrad Mathematical Society (he had turned seventy-six months earlier). It was an interesting talk with many details about his first steps in mathematics, including his discovery at a young age of the Euler-Maclaurin formula (recall that he taught himself almost entirely and never attended a high school).

${ }^{2}$ Zorya Yakovlevna Shapiro was then Gelfand's wife, with whom he wrote several important papers.
Our first paper was published in Uspekhi in 1973 in an issue honoring the seventieth birthday of Kolmogorov, and this was the beginning of my collaboration with Gelfand and Graev, which continued with some breaks over ten years. (At some point I will write more about this.) The first paper in this series (in Gelfand's opinion and mine, the best one) touched on many subjects that were important at that time. In particular, we discussed cohomology with coefficients in irreducible representations. In the next paper we described the cohomology of all semisimple groups without the Kazhdan property and gave explicit formulas for semisimple groups of rank 1. We also constructed irreducible nonlocal representations of the corresponding current groups. The next paper was devoted to representations of groups of diffeomorphisms and the start of the development of the geometry of configurations and its application to representation theory. We had no doubts (and it was later confirmed and reconfirmed) that this series of papers would have various applications and that the work would continue. In recent years M. I. Graev and I have found new constructions and new representations of functional groups, and this idea certainly has a future.

I would like to recall here one particular story. Our first paper had a natural continuation related to representations of groups of smooth functions on a manifold with values in a simple compact Lie group. The properties of these so-called "energy" representations depend on the dimension of the manifold.

This is not the place for details, but we were able to prove that the representations are reducible when the dimension is three or higher and when the dimension is two under some conditions on the length of a simple root (see our papers and also papers by R. Ismagilov, R. Hoeg-Krohn, S. Albaverio, and N. Wallach). Dimension one is special, and even at the beginning of our work, Gelfand said that we would probably have to deal with a von Neumann factor-representation. There were no obvious reasons or even preliminary estimates for this at that time; we had just started to work on the subject. After some years Gelfand's prediction was confirmed: in dimension one the energy representation is a factor-representation of type III1, and it turned out to be connected with a Wiener measure (not with the scalar one, but with a measure generated by Brownian motion on a compact group). Do you remember the mystic prediction by Gelfand fifty years ago that factors should connect with Wiener measures? Today the subject is actively being developed. 


\section{The Seminar}

Gelfand left us an enormous number of statements on different mathematical subjects. Someone with patience should collect them. Similarly, someone should have kept a diary of the Gelfand Seminar. Unfortunately, no one took the responsibility. Now we can pick up only bits and pieces. The seminars were like one-man shows, sometimes successful, sometimes rough, with salty humor, often relevant and instructive. I gave several talks at Gelfand Seminars in Moscow and later at Rutgers. I have already described my first very successful talk. The second was shorter and less successful but also distinctive. When I mentioned a not very interesting but very new fact, Gelfand called a graduate student to the blackboard and asked him to give a proof right away. Should I take this as an offense? Certainly not.

I attended the seminar for the first time when I was a graduate student. A French mathematician was talking about a paper by von Neumann. The talk was not very clear. Then Gelfand said, "Next time soand-so will give a talk on this paper" and mentioned several names of professors and graduate students and immediately started to evaluate the future talks. He said that one professor's talk would give an illusion of understanding, but in reality neither the speaker nor the listeners would have any idea of what was going on. Another one would speak about something else, including his own results, and so on. All this added some drama to the seminar. One could take it seriously or with a smile. Much later Gelfand told me that L. D. Landau ran a seminar in the style of Pauli (which bore a surface resemblance to Gelfand's but was somewhat more rude). Gelfand himself attended Landau's seminar and once repeated to me a joke made by M. Migdal: "Gelfand goes to the physicists as an intellectual to the peasantry." 3

At the same time, we must note that the Gelfand style was not for everyone. It drove some away and even complicated their lives. For example, one of his first, and most beloved, students was F. A. Berezin. At one point they parted ways completely. In the 1970s I tried unsuccessfully to get them to meet and talk. Nowadays, Berezin's work, especially in supermathematics, has gotten worldwide recognition, but he did not live to see this. Because I was not nearby and did not talk to Gelfand very often, I was able to avoid the frustration of some of his coauthors who worked with him more closely. On the other hand, with me he was always extremely polite and friendly.

\footnotetext{
${ }^{3}$ A reference to the political movement in Russia in the 1870 s known as "Going to the People" (Хождение к народу), during which intellectuals went to the countryside in expectations of "enlightening" the peasants.
}

His telegrams on my birthday were always very complimentary.

\section{Life in the USSR}

It is more and more difficult nowadays to explain to my Western colleagues, as well as young people in Russia, what academic life was like in the USSR, or even what other parts of life were like. For example, why did Gelfand, with all his achievements in science and contributions to classified state projects (which were considered exceptionally important), become a full member of the Soviet Academy of Science only after a shameful delay? Of course, one of the reasons is an official academic and even governmental anti-Semitism. But, for example, this feeling was not so strong among physicists. There were other reasons as well.

Once Gelfand told me, "The situation was quite simple for me in the beginning of the 1950s [the time of the infamous "fight with cosmopolitism", when Gelfand lost his position in Moscow State University]: only those who were really interested in mathematics became my students." It was true at that time (and also later) that it was better to have another advisor for one's career. But he still had quite a number of students. And here we face one of the most important and, in some ways, transcendental qualities of Gelfand: he could attract very different kinds of people. He surrounded himself with a whirlpool of established mathematicians, newcomers to the field, biologists, and others. His seminar was a place for meetings, corridor discussions, exchanges of news, opinions, and so on. It was not by chance that the famous "letter from 99 mathematicians" defending A. Esenin-Volpin ${ }^{5}$ was openly distributed for signatures at his seminar. It was met by authorities with resentment and fear at the highest levels. In a totalitarian state such as the USSR, only those who were trusted and tested by the state could be a magnet for people. For this reason, officials, including official mathematicians, did not like Gelfand and his circle, not just out of consideration of their origins but also by the principle of "either with us or against us".

"Of course, we live in a prison," Gelfand once told me during a conference outside Moscow in the late 1970s during lively discussions between our scientists and others visiting from the West. Still, he always refused to take any Samizdat books from me. People should write more about this.

Before any of his few visits abroad, Gelfand had to pass a number of unpleasant procedures, as did almost all Soviet people. My friends and I,

\footnotetext{
${ }^{4}$ A euphemism for an anti-Semitic government program.

${ }^{5}$ In 1969 Esenin-Volpin was thrown into a psychiatric "hospital" for political reasons.
} 
who were openly denied the right to travel abroad for any purpose, were for that reason spared the examinations by party commissions of our behavior and of our knowledge of party politics. These seemed to us unfortunate, but at the same time as rather a prestigious distinction.

At the end of the 1980s and the beginning of the 1990s the desire of many scientists to leave Russia (quite understandably, especially at that time) touched Gelfand as well. His Moscow seminar died soon after that. It was impossible to replace its leader. A relatively modest version of the seminar was reestablished at Rutgers. One can only guess what might have happened had Gelfand stayed in Moscow.

I met Gelfand in the U.S. several times. While mathematical life was different in the U.S., Gelfand found his place. The celebration of his ninetieth birthday at Harvard was perfectly organized and became a real scientific event with his brilliant talk.

I have never had any doubts that the name of I. M. Gelfand will be one of the icons of mathematics in the twentieth century, because the century was not just a century of outstanding achievements but also of new conceptions. I. M. Gelfand valued and created exactly this sort of mathematics.

\section{Bertram Kostant}

\section{M. Gelfand}

I first heard of I. M. Gelfand when I was a graduate student in Chicago in the early 1950s. At that time, Gelfand's paper "Normed rings" played a major role in the area of modern harmonic analysis, which was then very popular with students. My thesis advisor, Irving Segal, had arranged for me to spend the years 1953-1955 at the Institute for Advanced Study in Princeton. I already knew Chern and Weil from my years in Chicago, and at Princeton I became friendly with Lefschetz, Hermann Weyl, von Neumann, and Einstein. Certainly I considered Gelfand to be in the same class as these twentiethcentury math and physics luminaries, and I looked forward to meeting him as well.

My chance for this meeting came about when I was invited to a 1971 summer school in Budapest. The activities of the school were organized by Gelfand himself, and I believe this was the first time he had been given permission to attend a conference outside the Soviet Union. A large number of his students and colleagues came with him. It was an unforgettable experience for me to be a participant in one of his famous multihour seminars. Gelfand is a coauthor in a vast number

Bertram Kostant is professor emeritus at the Massachusetts Institute of Technology. His email address is kostant@math.mit.edu. of papers. No doubt many of these papers were outgrowths of these seminars. He seemed to have a very distinctive style of inspiring research by posing probing questions to potential collaborators and insisting on not letting go until there was some sort of resolution. I went to Budapest carrying a recently written paper on the spherical principal series. Gelfand requested that I submit the paper to the proceedings (Lie Groups and Their Representations) of the Budapest conference. Even though this paper was later (1990) to win the Steele Prize, it was probably a mistake for me to publish the paper in the proceedings of the Budapest conference. The initial publisher, Halsted Press, went out of business, and the book did not appear until 1974. When it finally did appear, many people reported that it was very hard to find.

In June 1972, responding to an invitation, I went to Moscow, accompanied by my wife, Ann, for a three-week stay. I have to say that I was deeply touched and happy to be the recipient of Gelfand's warmth, friendship, and respect. In his 1970 ICM report he had included me in a very small (5) group of people whom he said had made outstanding contributions to representation theory. In Moscow, Gelfand made arrangements for me to address a meeting of the Moscow Mathematics Society, chaired by Shafarevich, with whom I had a pleasant lunch. Gelfand also made arrangements for me to meet on a regular basis with a number of his students, including Kazhdan, Bernstein, Kirillov, Gindikin, and his son, Sergei. I was also introduced to his colleagues Manin, Novikov, and Graev, and I spent an afternoon with Berezin in Gorky Park talking about (from my perspective, geometric) quantization.

One of the mathematical topics that came up during my conversations with Gelfand was the results in the first of the BGG, [BGG-71] papers. Somewhat earlier I had solved a problem of Bott which asked for a determination of the polynomial functions on the dual of a Cartan subalgebra which mapped, via Borel transgression, onto the dual basis of the Schubert classes of a general flag manifold. Acknowledging my precedence in solving this problem, [BGG-71] made a penetrating further development in the solution of this problem by introducing the very important BGG operators.

What was apparent to me during my stay was that Gelfand seemed to have created an environment where he was involved with both the personal as well as the professional lives of the many people around him. Gelfand's apartment was like Grand Central Station, with any number of people going in and out. He seemed to be carrying on $n$ conversations. I can't speak for the people orbiting around him, but as an outsider looking in I was charmed. Doing mathematics for me has 
had many lonely moments, but in this exciting environment I sensed that mathematics (like much of physics) could be done in a communal, socially satisfying way.

There are any number of unforgettable incidents which occurred during my Moscow visit. The following was very embarrassing for me (but not without its comic aspects). One day Gelfand invited Ann and me to have some borscht with him at the restaurant of the Soviet Academy. All the tables in the room were filled with people, except for the table opposite us. That table was occupied by only one man. Suddenly Gelfand started whispering to me, but I couldn't make out what he was saying. Then he repeated it somewhat louder. Finally, when I heard what he was saying, I foolishly blurted out loud the name Lysenko. The room became still, and then I realized what I had done. "Oh my God," I thought. "I have gotten Gelfand into trouble with the authorities." For a moment visions of him being dragged off to the Gulag went through my head. But all turned out well. By the early seventies Lysenko had apparently been defanged.

Much later Ann and I saw a great deal of Gelfand when he was invited to Cambridge, Massachusetts, in the United States to receive an honorary degree from Harvard. A number of scenes during that visit still stick in my head. One was at my house during a party as I watched him on the floor with a bunch of young kids, somehow managing to keep their interest by telling them some mathematical gems. Another was the scene at the breakfast table after Gelfand spent the night in our house. Gelfand was complaining about the absence of good bread in the U.S. and the difficulty of finding healthy food. I countered by pointing to the Swiss muesli in my breakfast bowl. Exhibiting a sample of his delicious (no pun intended) sense of humor, he then proceeded to eat the fruits and raisins in my bowl, all the while cautioning me not to have that for breakfast because it wasn't good for me.

Sometime during his visit I met him in New York City and introduced him to my son, Steven, then engaged in filmmaking. Gelfand gave a long discourse to Steven and his friends on Stanislavski. Apparently method acting was one of Gelfand's many artistic interests. I also took him to see Jean Renoir's famous film Le Grande Illusion, whose main theme was the disintegration of European aristocracy in the wake of World War I. I was totally surprised by his reaction to the film. Since it was made in the late thirties, he thought it was unconscionable that a prominent film was produced on such a topic at a time when Hitler was planning his march across Europe.

Another scene that sticks in my head was when, while driving Gelfand to the airport for his flight back to Russia, he started to recite poems of Ossip Mandelstam, Anna Akhmatova, and others. I remember recording him on tape, but unfortunately I seem to have misplaced the tape.

Several years later my mathematical interests intersected with those of Gelfand in the area of completely integrable systems. In 1979 I had written a paper showing that the complete integrability of the open Toda lattice arises from a consideration of a certain coadjoint orbit of the Borel subgroup. Gelfand brilliantly put this result in an infinitedimensional context and applied it to the theory of pseudodifferential operators. I believe a

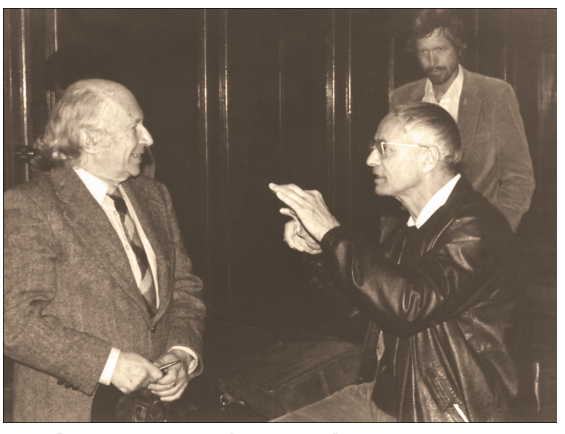

With Serre and MacPherson at Monday night seminar, Moscow State University, 1984.

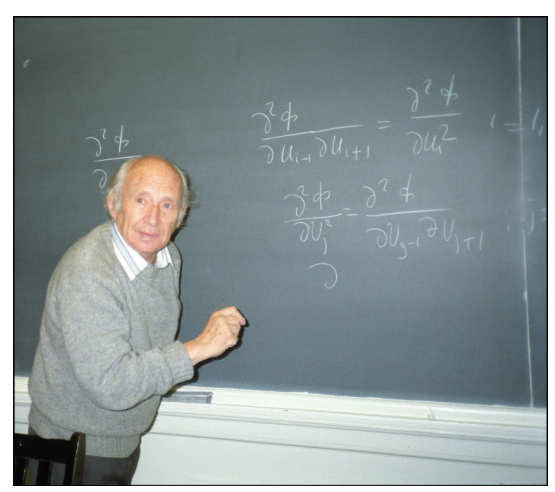

Lecture at MIT, 1990. similar observation was made by Mark Adler.

I would like to end here by citing a mathematically philosophical statement of Gelfand which I think deserves considerable attention. It also opens a little window, presenting us with a view of the way Gelfand's mind sometimes worked. One of my first papers gave a formula for the multiplicity of a weight in finite-dimensional (Cartan-Weyl) representation theory. A key ingredient of the formula was the introduction of a partition function on the positive part of the root lattice. The partition function was very easy to define combinatorially, but giving an expression for its value at a particular lattice point was altogether a different matter. Gelfand was very interested in this partition function and mentioned it on many occasions. He finally convinced himself that no algebraic formula existed which would give its values everywhere. He dealt with this realization as follows. One day he said to me that in any good mathematical theory there should be at least one "transcendental" element and this transcendental element should account for many of the subtleties of the theory. In the Cartan-Weyl theory, he said that my partition function was the transcendental element. 


\section{Simon Gindikin}

\section{Years of Gelfand's Integral Geometry}

As I begin this paper, the entire fifty-year history of integral geometry in Gelfand's life unfolds before my eyes. I was fortunate enough to collaborate with him during some key moments. I would like to discuss here those points that appear to me to be the most important in this wonderful endeavor.

\section{First Presentation}

I remember well a meeting of Dynkin's seminar on Lie groups, most likely in the spring of 1959. The seminar, which had been only for undergraduate students at first, at this point combined both undergraduate students (Kirillov, Vinberg, me) and well-known mathematicians (Karpelevich, Berezin, Piatetski-Shapiro). Suddenly, Gelfand appeared (late as usual and accompanied by Graev), and with enormous enthusiasm he began talking about their new work [1]. This was the first time their work on integral geometry was presented.

It may appear strange that Gelfand did not present this in his own seminar (in fact, he almost never presented his new results there) and that he selected what was mostly a student seminar. Without a doubt, this was no accident, since Gelfand was always very precise in selecting venues for his talks, and this fit well into traditions of Moscow's mathematical life. My mind's eye does not see any specifics of the presentation but rather recalls Gelfand's excitement, obvious to the listeners, and his certainty that something significant had opened before him-a new direction of geometric analysis, which he proposed to call "integral geometry", as it was equaled in importance only by differential geometry. He commented that Blaschke used this term for a certain class of problems connected with calculations of geometric measures but that this narrow area did not deserve such an ambitious title, and so he felt justified in appropriating it for this new field. The contentious nature of such a position is obvious. Such things worried Gelfand only a little, and I will not venture an opinion on the subject. I think this is important for understanding Gelfand's emotional state. He reminded us of his frequent saying that "representation theory is all of mathematics." (I heard this many times, and Manin once recalled that he also heard from Gelfand that "all mathematics is representation theory," noting the delicate difference between these aphorisms.) From now on, Gelfand said, he considered that "integral geometry is all of mathematics."

Simon Gindikin is Board of Governors Professor at Rutgers University. His email address is gindikin@math. rutgers. edu.
Now a word about Gelfand's mathematical motivation. He considered that the main problem of the theory of representations was the decomposition of certain reducible representations-most importantly, regular representations on homogeneous spaces-into a sum (maybe continuous) of irreducible representations (Plancherel formulas). The class of groups and homogeneous spaces was not specified in the talk. It was only clear that complex semisimple Lie groups, together with their homogeneous spaces with maximally compact and Cartan isotropy subgroups, were in this class. The first two spaces are symmetric, but the third is not. This is of principal importance-spaces that are not symmetric are considered. From the very beginning the decomposition into irreducible representations is interpreted as a noncommutative analog of the Fourier integral: under the conditions of a continuous simple spectrum, we can talk about the "projections" of a function on a homogeneous space onto irreducible representations. The usual Fourier transform on $\mathbb{R}^{n}$ has a geometric twin in the Radon transform: integration on hyperplanes. They are connected with each other via the one-dimensional Fourier transform. The marvelous discovery of Gelfand-Graev was that, in the case of semisimple Lie groups, there is an analogous geometric twin: on homogeneous spaces we consider horospheres-the set of orbits of all maximally unipotent subgroups-and the horospherical transform (the operator of integration on horospheres). The generalized Fourier transform and the horospherical transform are connected by the (commutative) Mellin transform, equivalently, with the commutative Fourier transform. For this reason, problems about the Plancherel formula and inversion of the horospherical transform on a homogeneous space are trivially reduced to each other. Horospheres in hyperbolic geometry were well known (and highly valued) already by their creators: they are spheres of infinite radius with centers at infinity and different from hyperbolic hyperplanes. For other homogeneous spaces (including symmetric spaces), they had remained unnoticed before the work of Gelfand-Graev.

A posteriori, the idea of the horospherical transform seems almost obvious. Let $G$ be a complex semisimple Lie group and let $B=M A N$ be a Borel subgroup; here $N$ is the nilpotent radical, $M A$ is a Levi subgroup (which is commutative in this case) with $M$ and $A$ its compact and vector subgroups, respectively. Then the manifold of horospheres is $\Xi=G / N$. Gelfand and Graev called it the "principal affine space". On this space there are two commuting actions: that of the group $G$ acting by left shifts and that of the group $M A$ acting by right shifts; the latter action is well defined because $M A$ normalizes $N$. 
The decomposition of the regular representation on $\Xi$ relative to the action of $M A$ gives precisely the irreducible representations of group $G$ realized in the spaces of sections of line bundles over the flag manifold $F=G / B$ (the "principal projective space" in Gelfand-Graev's terminology). This is simply a rephrasing of the conventional realization of the principal series representations. Therefore, if the horospherical transform (which is an intertwining operator) is injective, the decomposition of the regular representation is reduced to the decomposition relative to the action of $M A$ on the space of horospheres and the above-mentioned Mellin transform along the abelian subgroup $A$.

\section{Prehistory}

Needless to say, the real road to the discovery just outlined was entirely different. I was lucky to hear about it from Gelfand, and I want to share it here. Three papers appeared in 1947-by Bargmann, Gelfand-Naimark, and Harish-Chandra-in which unitary representations of the Lorentz group were found. I believe that I once heard Gelfand's observation that important things in mathematics spring forward independently from three places at once (the history of mathematics indeed delivers striking examples of this, beginning with non-Euclidean geometry). The Lorentz group is locally isomorphic to group $S L(2 ; \mathbb{C})$. Not surprisingly, the initiative in this problem belonged to the physicists, and the authors were targeting physical applications. (Bargmann was solving Pauli's problem and discussed it with Wigner and von Neumann; the problem was suggested to Harish-Chandra by Dirac; the first publication of Gelfand-Naimark was in a physics journal.) Because representations of the rotation group play a key role in nonrelativistic quantum mechanics, it was natural to expect representations of the Lorentz group to play an analogous role in the relativistic case. The difficulty lay in the fact that, while the rotation group is compact and all its irreducible unitary representations are finite dimensional, the Lorentz group is not compact and its only finitedimensional unitary representation is the trivial one-dimensional representation. (It is precisely the unitary representations that have a physical interpretation.)

In the 1930s Dirac and Wigner considered certain infinite-dimensional unitary representations of the Lorentz group. From the other direction, in the early 1940s Gelfand-Raikov constructed an analog to the theory of Peter-Weyl for general locally compact groups using infinite-dimensional unitary representations. It seems likely that there was a consensus that the description of all unitary representations of the Lorentz group could not be done in an explicit form and had to include difficult considerations of von Neumann factors. Doubtless the biggest surprise for the authors was how explicitly and simply all irreducible unitary representations of the Lorentz group can be described.

Bargmann also

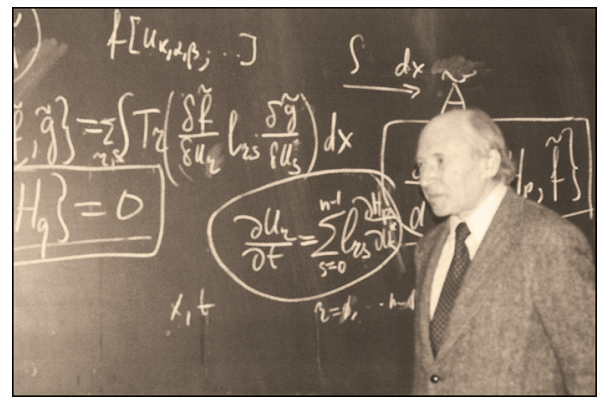

considered representations of the "real" Lorentz group $S L(2 ; \mathbb{R})$ and discovered representations of the discrete series, realized in holomorphic functions on the disk. The central result is the completeness of the constructed unitary representations, although Bargmann and HarishChandra proved only an infinitesimal version of the completeness. Bargmann also proved a certain statement of the completeness of matrix coefficients.

At the same time, Gelfand and Naimark [2] went much further, establishing essentially all the principal concepts of the theory of unitary representations. They introduced characters of irreducible unitary representations as distributions, showed that characters define representations up to equivalence, and computed explicitly the character values on regular elements, thus obtaining an exact analog of the classical Weyl character formula. The paper contains a complicated analytical proof of the fact that their list of irreducible unitary representations is complete; however, the focus of the work is an analog of the Plancherel formula. This provides the (continuous) decomposition into irreducibles of the two-sided regular representation on the $L^{2}$ space of the group relative to the invariant measure.

It turned out that in this decomposition only some unitary representations appear; they were called representatives of the principal series (the rest of the unitary representations make up the complementary series). It is remarkable that the Plancherel measure, which appears in the expression of a norm of a function on a group through the norms of its projections on irreducible components, was computed explicitly. However, this remarkable formula, reminiscent of the classical Weyl formula for finite-dimensional representations, was a result of almost ten pages of dense computations without any visible attempts to uncover the conceptual structure. Later this was pointed out by Mautner while refereeing a more conceptual proof found by Harish-Chandra. This fitted the style of Gelfand, who at the beginning was more concerned with the beauty and clarity of the final result than with a simplification of the proof. 


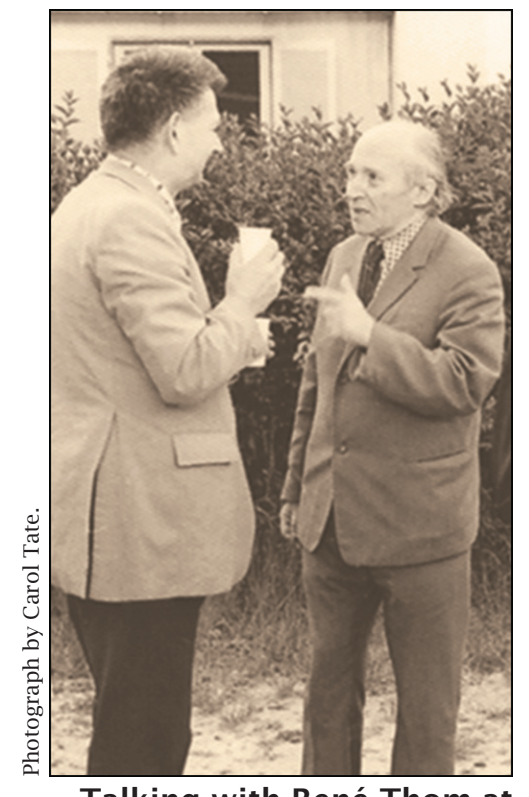

be nothing short of heroic IHÉS, 1974. of explicit formulas through intense analytic attacks, overcoming many obstacles. The proof of an analog of the Plancherel formula, the peak of the theory, especially stands out. The authors were unable to generalize an already difficult proof from the paper about the Lorentz group; the proof given in the book is exceptionally difficult and contains many brilliant inventions. Gelfand especially prized the calculations in a certain generalization of elliptic coordinates.

Around 1950 significant changes took place in Gelfand's mathematical life. First of all, he concluded his extraordinarily fruitful collaboration with Naimark. More than that, he decisively changed the organization of his work. If before, he concentrated on some one direction (Banach spaces, Banach algebras, theory of representations) at every moment, now he worked simultaneously with several coauthors on problems from very different areas. He did not abandon the theory of representations, and for many years Graev became his primary collaborator. Soon Harish-Chandra gave a conceptual proof of the Plancherel formula for all complex semisimple Lie groups and also for $S L(2 ; \mathbb{R})$. However, something kept Gelfand from being satisfied, and from time to time he continued returning to the Plancherel formula. In 1953 he and Graev offered an exceptionally elegant way to derive it through application of the result of M. Riesz about regularization of powers of a quadratic form.

Gelfand kept coming back to his proof of the Plancherel formula for the Lorentz group, which had not been generalized to other groups and in which he felt something important was left not understood. In 1958 suddenly he saw in this proof something that had remained hidden for almost ten years, namely, that a large part of the proof was dedicated to the solution of the following elementary-sounding problem of geometrical analysis: Consider a function $f(\alpha, \beta, \delta)$ of three complex variables. Integrate this function (in the real sense) on all complex lines intersecting the hyperbola $\alpha=\lambda, \delta=\lambda^{-1}, \beta=0$, where $\lambda \in \mathbb{C}$. Then reconstruct $f$ through all of these integrals. What connection do these lines have to the Lorentz group $S L(2 ; \mathbb{C})$ ? Consider the set of horocycles in this group: the two-sided shifts of the unipotent subgroup $N$ of unit upper triangular matrices $\left(\begin{array}{ll}1 & u \\ 0 & 1\end{array}\right)$ with $u \in \mathbb{C}$. This set turns out to be exactly the three-parameter family (on $\mathbb{C}$ ) of all (complex) lines in the group, considered as a hyperboloid $\alpha \delta-\beta \gamma=1$ in $\mathbb{C}^{4}$. If we project the hyperboloid onto the plane with coordinates $(\alpha, \beta, \delta)$, then almost all the horocycles (excluding those that project into points) transform into lines intersecting the hyperbola. The solution to the inversion problem in [1] is a very simple formula, reminiscent of the Radon inversion formula. For the inverse of the generalized Fourier transform on the group (Plancherel formula), it remains only to invert the usual Mellin transform. After this interpretation, it was straightforward for Gelfand and Graev to define horospheres and the horospherical transform in the general case. The authors of [2] apparently did not know of this geometrical interpretation, but looking at the paper, one can't stop marveling at how closely they followed it in their calculations (the corresponding text about this problem of integral geometry in the fifth volume of Generalized Functions [4] differs from the text of the 1947 paper only in the addition of certain words without any substantial changes to any of the formulas).

However, at this point the romantic side of the story concludes and prosaic everyday mathematical problems begin. There is no doubt about the beauty of the horospherical transform and its significance, but it is fair to raise the question: what does it contribute to the theory of representations? From the very beginning, Gelfand had the idea that there have to be direct methods to invert certain generalized Radon transforms, including the horospherical transforms, and there had to be a more natural way to derive Plancherel formulas. However, in 1959 such a way was not uncovered, and in [1] the situation is essentially the opposite: the formula for the inversion of the horospherical transform for complex semisimple Lie groups and certain homogeneous spaces was derived from the already-known Plancherel formula. A remarkable formula was produced, which resembled the the Radon inversion formula in odd-dimensional spaces but which also showed which modifications 
are required for spaces of rank greater than 1: one needed to average (over the set of horospheres passing through a point) the result of applying an explicit differential operator (of the order equal to the rank) that acts along parallel horospheres. It remained to develop direct methods of integral geometry and to understand what advantages this approach had to harmonic analysis. This was accomplished, partially, only ten years later [5].

Even so, some important supporting observations slowly accumulated. Gelfand noticed in the very beginning that the symmetric Riemannian space for the group $S L(2 ; \mathbb{C})$ is the three-dimensional hyperbolic space, with horospheres in the classical sense. It turned out that the inversion formula for this horospherical transform, with the appropriate definitions, exactly matches the formula for the inversion of the Radon transform in three-dimensional Euclidean space. By contrast, the Plancherel formulas for the Fourier transform in Euclidean and hyperbolic spaces differ dramatically. In a sense, the horospherical transform is independent of curvature! There was no doubt of the importance of this observation, but for a surprisingly long time there were no attempts to incorporate this into some general result. Today it is clear that if one considers for a Riemannian symmetric space of negative curvature its "flat" twin (sending curvature to zero), then the inversion formulas for the horospherical transforms will be identical for these two spaces. It seems to me that this explains the nature of the simple explicit formulas for the representations of semisimple Lie groups (which surprised their creators): from the point of view of the horospherical approach, the problems turn out to be equivalent to flat ones. There exists another important circumstance which doubtlessly was tacitly understood, even though I've never encountered a discussion of it. Namely, in the standard approach to the Plancherel formula, integrals on conjugacy classes are regularized so as to be meaningful as "integrals" on singular conjugacy classes, eventually, on the unit class. Horospheres, in a certain sense, are generators of conjugacy classes; bringing them into the analysis illuminates the consideration of singular classes.

\section{Integral Geometry of Lines and Curves}

The biggest success in the following period was connected with the interpretation of horocycles on the Lorentz group as lines intersecting a hyperbola. Everything began with a natural question: What happens if the hyperbola is replaced by another curve? Kirillov proved that under such a replacement the inversion formula remains unchanged. This meant something very important: with a replacement of the hyperbola by another curve, the group disappeared, and it became clear that a significant part of the theory of representations of the Lorentz group is connected not with the group structure, but rather with some sort of more general geometrical structure. This was the first supporting evidence for Gelfand's project to embed the theory of representations into a wider area of geometrical analysis.

The next step was again evident: understand the nature of the condition on a family of lines "to intersect a fixed curve". The family of all lines in $\mathbb{C}^{3}$ depends on four (complex) parameters. For integral geometry it is natural to consider families with three parameters (complexes of lines in classical terminology), because then integration transforms a function of three variables into a function of three variables. For which complexes of lines are there inversion formulas of Radon's type? Gelfand and Graev [4] showed that this is possible not only for complexes of lines intersecting a fixed curve but also for complexes of lines tangent to a fixed surface and not for any others. They called such complexes admissible. It is worthwhile to say a few words about the proof of this astounding result.

F. John discovered that in the real case the image of the operator of integration along lines in a three-dimensional space is described by the ultrahyperbolic differential equation. In the complex case one has to consider the holomorphic and antiholomorphic versions of this differential equation. It turns out that existence of the required inversion formula is equivalent to solvability of the Goursat problem and therefore coincides with the characteristic condition for this operator. This is a nonlinear equation that can be integrated using the method of Hamilton-Jacobi. The consideration of bicharacteristics gives the description of admissible complexes. These complexes had already appeared in classical differential geometry. They are, in a natural sense, maximally degenerate: the lines of the complex that intersect one fixed line of the complex lie (infinitesimally) in one plane.

This result is generalized to complexes of lines in general position in spaces of any dimension. The natural next step is to consider the possibility of the replacement of lines by curves. The final result in this direction was obtained by Bernstein and me [12] (after Gelfand, Shapiro, and I previously found a universal structure of inversion formulas for complexes of curves [6]). Admissible complexes of curves turned out to be exactly infinitesimally full families of rational curves (infinitesimally, they are spaces of all sections of a certain vector bundle on the projected line). Here the most significant condition is for the curves to be simultaneously rational. This is a quite effective condition, allowing the construction of many explicit examples of families of curves with inversion formulas of Radon type, for example, curves of the second 
order. In these constructions it is natural to give up the requirement that the family of curves be a complex (the number of parameters of the family equals the dimension of the manifold). In this case, the possibility exists to consider a certain generalization of a problem of integral geometry, but more instructive are the possibilities of applications beyond the theory of representations. There exists, for example, a connection with the twistor theory of Penrose: the four-parameter admissible family of curves corresponds to conformal right-flat four-metrics (the self-dual part of Weyl curvature equals zero). This allowed us to develop a method of construction of explicit solutions of the Einstein self-dual equation. It seems to me that, in the case of curves, integral geometry has been successfully fully developed. It appears to be some part of nonlinear analysis, with a focus on explicit integrable problems, and it is connected with the method of the inverse problem for the case of one spectral parameter.

\section{Complexes of Planes}

The situation with submanifolds of dimension higher than one is significantly more complicated, and there may be no opportunity in this case for work to advance as far. It appears that it is similar to the possibility of the integrability in inverse problems with several spectral parameters. However, a few essential results have been achieved. Let us recall that beginning with the first work on integral geometry [1], the grand challenge was to find a proof of the Plancherel formula through inversion of the horospherical transform. Ten years passed before Gelfand, Graev, and Shapiro did this [6], [5] for the group $S L(n ; \mathbb{C})$. We have already discussed the fact that when $n=2$, horospheres can be considered as lines. For arbitrary $n$, horospheres for this group can also be considered as planes of dimension $k=n(n-1) / 2$ in $\mathbb{C}^{N}$, where $N=n^{2}-1$. The dimension of this family of planes coincides with $N$, so we have a complex of $k$-planes. The key idea is to study the problem of reconstructing a function through its integrals on planes from an arbitrary complex, not limiting ourselves to a complex of horospheres. Let us note that the problem of reconstructing a function through its integrals on all planes is extremely overdetermined and the transition to complexes is a natural way to make the problem well defined. It turns out that for reconstruction at a point it is always possible to write a formula reminiscent of the Radon inversion formula. This formula involves averaging a certain explicit differential operator (of order $2 k$ ) along the set of planes of the complex which pass through a point. This operator is defined on the set of all $k$-planes; however, we can compute it using its restriction to the set of planes of the complex only under very strong conditions. Let us call complexes satisfying these conditions admissible. There again arises a certain condition involving characteristics. We can describe the situation slightly differently. The image of the operator of integration over $k$-planes is described via a certain system of differential equations of the second order, generalizing the John equation, and admissible complexes must be characteristic for this system. In some sense, the admissible complexes are maximally degenerate.

The nonlinear problem of the description of admissible complexes is unlikely to be integrable for $k>1$. However, Gelfand and Graev [5] checked directly that the complex of horospheres in $S L(n ; \mathbb{C})$ is admissible. As a result, we get an inversion of the horospherical transform and as a consequence the Plancherel formula. I am certain that there is something very significant in the degeneracy of the manifold of horospheres. I think that the geometric background of the theory of representations of semisimple Lie groups lies in this phenomenon. Clearly, this result makes substantial use of the possibility of considering horospheres on $S L(n ; \mathbb{C})$ as planes. For other semisimple Lie groups this is impossible, but I showed that the construction described above may be generalized for these groups by considering admissible complexes of arbitrary submanifolds [8].

\section{Nonlocal Problems and Integral Geometry for Discrete Series}

In the case of complex groups the structure of inversion formulas with averaging of a differential operator means that, in particular, these formulas are local: for reconstruction of the function at a point, it is sufficient to know the integrals on the horospheres close to this point. In the case of the Radon transform, this is the case only in odddimensional spaces. In even-dimensional spaces the inversion formula is nonlocal: in this situation a pseudodifferential operator is averaged. If we were to go from complex groups to real ones and their homogeneous spaces, the first new difficulty would be connected with the inevitable appearance of nonlocal formulas. It is natural to begin with Riemannian symmetric spaces of noncompact type $X=G / K$, where $G$ is a real semisimple Lie group and $K$ is a maximally compact subgroup of $G$. Then the horospherical transform is always injective, and the inversion formula is local if and only if the multiplicities of all roots are even. In this case the inversion formula can be derived using the method described above for complex groups. However, the general case must involve nonlocal inversion formulas and requires new ideas. After Karpelevich and I computed the Plancherel measure on these spaces through the product 
formula for the Harish-Chandra $c$-function, we found [13] in this case the analog of the approach of Gelfand-Graev to complex groups in their first paper [1]: we computed the kernel of the inverse horospherical transform as the inverse Mellin transform of the Plancherel density. We made this calculation for classical groups. Later, Beerends made it in the general case. The next step should be a direct derivation of these inversion formulas using methods of geometric analysis (as in the case of even multiplicities), but so far this has not been done. We tried with Gelfand [9] to move in this direction, developing a certain symmetric analog of differential forms on real Grassmannians, which doubtless has its own interest. However, we did not achieve significant progress in this problem. It seems to me that today many points look clearer, and this problem looks realistically solvable.

Finally, we can begin discussing the main obstacle to the development of the theory of representations on the basis of the horospherical transform, which was clear from the very beginning. For real semisimple Lie groups the horospherical transform as a rule has a kernel, which consists of all of its representation series except for the maximally continuous ones. This is already true for the group $S L(2 ; \mathbb{R})$ : if we decompose its regular representation into the sum of subspaces $L_{c}, L_{d}$ corresponding to continuous and discrete series respectively, then the kernel of the horospherical transform coincides with $L_{d}$ and the image is isomorphic to $L_{c}$. There does not appear to be a way to invert the horospherical transform or to derive in this way the Plancherel formula. It is easy to interpret this as a dramatic limitation of the power of integral geometry in the case of real groups, where discrete series play a central role. This appears to be the reason that Gelfand's idea of applying integral geometry to the theory of representations has not evoked much enthusiasm amongst experts on representation theory.

I can be a witness to the fact that Gelfand never believed that the area of applications of horospheres is bounded by continuous series. In his opinion it was necessary to understand what corresponds to horospheres in the case of discrete series, and this is perfectly possible. This resonated with his faith in the aesthetic harmony of mathematics. In his first talk, which it befell me to hear (in 1955), he said about von Neumann factors of the second type (which did not have any known applications at that time): "Such beauty must not vanish!" Only in one case-an imaginary threedimensional hyperbolic space-did Gelfand and Graev manage [7] to find an appropriate expansion: the discrete series there was connected with certain degenerate horospheres. However, this was connected with certain very special circumstances, and there was no chance of direct generalization.

The case of $S L(2 ; \mathbb{R})$ remained the first call to action. In 1977 Gelfand and I attempted to understand it [10]. The philosophy of our approach was the fact that the Plancherel formula has to be derived in two stages. First, one has to find projections into subspaces corresponding to the representation series. Series of representations correspond to equivalence classes of Cartan subgroups. The decomposition of each series into irreducible subspaces is reduced to a commutative Fourier transform (continuous or discrete) corresponding to the associated Cartan subgroup. So the first stage is the princi-

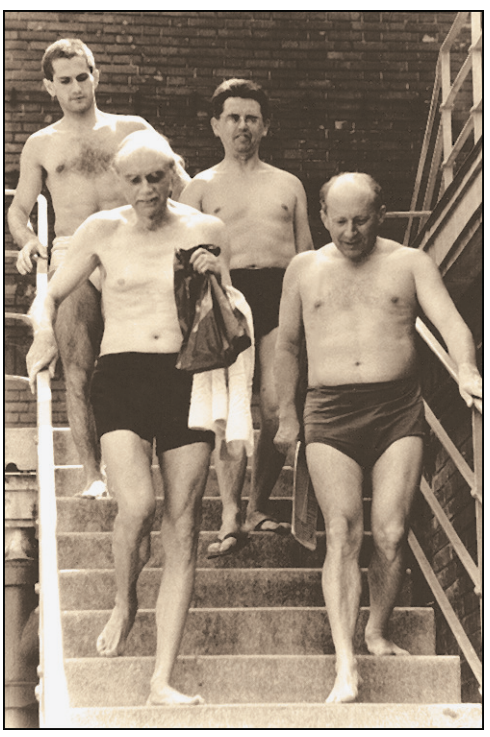

Budapest swimming pool, 1966. First row, P. Dirac, I. Gelfand; second row, B. Bollobas, M. Arato. pal one. Two of the main connected problems are the finding of projections into the series and the internal analytic characterization of subspaces for the series. We solved these problems for $S L(2 ; \mathbb{R})$. Subspaces corresponding to holomorphic and antiholomorphic discrete series can be characterized as boundary values on $S L(2 ; \mathbb{R})$ of holomorphic functions in certain tube domains in the complex group $S L(2 ; \mathbb{C})$. Projections may be interpreted as certain analogs of the Cauchy integral formula. I remember how happy Gelfand was with this result. He often said that new, significant things in representations have to be already nontrivial for $S L(2)$. We expected that in the general case as well the series would be connected with certain tube domains, which may not be Stein manifolds, and then it would be required to consider $\bar{\partial}$ cohomology on these domains. Later this was called the Gelfand-Gindikin program. Significant progress was achieved in this program, but only for holomorphic discrete series.

It could have been expected that the projections into the series had to be somehow connected with integral geometry, but at the time it proved impossible to find an appropriate generalization of the horospheres. Much later I discovered a certain natural construction [14]. On $G=S L(2 ; \mathbb{C})$ there are two classes of horospheres, each of which allows the possibility of constructing a horospherical transform. We have discussed the one-dimensional horocycles. However, it is also possible to consider two-dimensional horospheres-orbits of maximal unipotent groups in $S L(2 ; \mathbb{C}) \times S L(2 ; \mathbb{C})$ under the 
two-sided action. Their geometrical characterization consists of sections of the group, considered as a hyperboloid, by isotropic planes. Horocycles are linear generators of two-dimensional horospheres; for this reason, both versions of the horospherical transform are equivalent. For $S L(2 ; \mathbb{R})$ both transforms have kernels. The idea is that since there are not enough real horospheres, we must consider certain complex ones.

Let us consider those complex horospheres which do not intersect the real subgroup $S L(2 ; \mathbb{R})$. There are three types of such horospheres. Instead of integration on real horospheres, we consider the convolution (on the real group) of Cauchy kernels with singularities on the complex horospheres without real points. As a result, the horospherical transform of their three components is defined. It already does not have a kernel, and images of the various components can be decomposed using representations of various series. The inversion of the horospherical representation gives projectors onto series and then also the Plancherel formula. At the same time, the continuation of functions from the continuous series is obtained as a one-dimensional $\bar{\partial}$-cohomology in a certain tube domain, in agreement with our hypothesis. It is my hope that the correct development of the understanding of complex horospheres for real semisimple Lie groups is sufficient for the construction of the integral-geometrical equivalent of the theory of representations. Interestingly, this can be done even for compact Lie groups, for which there are no real horospheres at all.

Gelfand (in collaboration with Graev and me) worked on many other aspects of integral geometry. I have not discussed these results because I wanted to concentrate here on integral geometry connecting with the theory of representations, which seems to me to be the most important part of this project. It is intriguing that Gelfand was interested in the Radon transform long before its connection with the theory of representations was discovered. He liked to offer problems concerning the Radon transform to students, though he never worked in this direction himself. This was almost a premonition of future connections, an ability often shown by exceptional mathematicians and typical for him. Integral geometry was not one of his most successful projects. It did not gain him broad recognition or a multitude of followers. Yet we find in it, in a very pronounced way, his inimitable approach to mathematics. Like every other mathematician lucky enough to have worked with Gelfand, I have gotten used to trusting his extraordinary intuition, and I am certain that the story is not over. Perhaps I will see the realization of his fantastic project yet.

\section{References}

[1] I. M. GELFAND, M. I. GRAEV, Geometry of homogeneous spaces, representations of groups in homogeneous spaces and related questions of integral geometry, Amer. Math. Soc. Transl. (2), 37 (1964), pp. 351-429.

[2] I. M. GELFAND, M. A. NAIMARK, Unitary representations of the Lorentz group, Izv. Acad. Nauk SSSR, Ser. Mat., 11 (1947), pp. 411-504.

[3] I. M. GELFAND, Integral geometry and its relation to the theory of group representations, Russ. Math. Surv., 15 (1960), no. 2, pp. 143-151.

[4] I. M. GelFAnd, M. I. Graev, N. YA. Vilenkin, Generalized Functions, Vol. 5. Integral Geometry and Representation Theory, Academic Press, 1966.

[5] I. M. Gelfand, M. I. GRAEV, Complexes of $k$ dimensional planes in the space $\mathbb{C}^{n}$ and Plancherel's formula for the group $G L(n, \mathbb{C})$, Soviet Math. Dokl., 9 (1968), pp. 394-398.

[6] I. M. GELFAND, M. I. GRAEV, Z. YA. Shapiro, Integral geometry on $k$-dimensional planes, Funct. Anal. Appl., 1 (1967), no. 1, pp. 14-27.

[7] I. M. GELFAND, M. I. GRAEV, An application of the horospheres method to the spectral analysis of functions in real and imaginary Lobachevsky space, Trudi Mosk. Math. Soc., 11 (1962), pp. 243-308.

[8] S. G. GINDIKIN, Integral geometry on symmetric manifolds, Amer. Math. Soc. Transl. (2), 148 (1991), pp. 29-37.

[9] I. M. GELFAND, S. G. GINDIKIN, Nonlocal inversion formulas in real integral geometry, Funct. Anal. Appl., 11 (1977), pp. 173-179.

[10] _ Complex manifolds whose skeletons are semisimple real Lie groups and holomorphic discrete series of representations, Funct. Anal. Appl., 11 (1977), pp. 258-265.

[11] I. M. GELFAnd, S. G. Gindikin, Z. YA. Shapiro, The local problem of integral geometry in the space of curves, Funct. Anal. Appl., 13 (1979), pp. 87-102.

[12] J. BERNSTEIN, S. GiNDIKIN, Notes on integral geometry for manifolds of curves, Amer. Math. Soc. Transl. (2), 210 (2003), pp. 57-80.

[13] S. G. Gindikin, F. I. KARPELEVICH, A problem of integral geometry, Selecta Math. Sov., 1 (1981), pp. 160-184.

[14] S. GINDIKIN, Integral geometry on $S L(2 ; \mathbb{R})$, Math. Res. Lett., 7 (2000), pp. 417-432.

\section{Peter Lax}

\section{M. Gelfand}

Like everyone else, I first heard Gelfand's name as the author of the famous theorem on maximal ideals in commutative Banach algebras and its application to prove Wiener's theorem about functions with absolutely convergent Fourier series. The following story describes the reception of this result in the U.S.

Shortly after its publication, Ralph Phillips presented this result at Harvard. It created such a

Peter Lax is professor emeritus at the Courant Institute of Mathematical Sciences. His email address is 1ax@courant.nyu.edu. 
stir that he was asked to repeat the lecture, this time to the whole faculty. And then he was asked to present it a third time to G. D. Birkhoff alone.

Other early basic results were the Gelfand formula for the spectral radius and the Gelfand-Levitan inverse spectral theory for ordinary differential operators. If you have a completely integrable system, you are supposed to be able to integrate it completely. For the KdV equation, the landmark result of Gelfand and Levitan on the inverse spectral problem for second-order ODEs turned out to be the key to perform the integration.

It is a measure of Gelfand's lifetime achievements that these spectacular early results are viewed today as merely a small part of his total work.

Here is a story that sheds light on Gelfand's modesty. I was one of three members of a committee to award the first Wolf Prize. We all agreed that the prize should be given to the greatest living mathematician. That person, in my opinion, was Carl Ludwig Siegel, but another member of the committee insisted that it was Israel Moiseevich Gelfand. So we argued back and forth fruitlessly until we decided to divide the prize. Some time later Gelfand remarked in a chance conversation, "It was a great honor for me to share a prize with Siegel.”

The world shall not see the like of Israel M. Gelfand for a long, long time.

\section{Andrei Zelevinsky}

\section{Remembering I. M. Gelfand}

On December 6, 2009, two months after I. M. Gelfand passed away, Rutgers University, his last place of work, held a Gelfand Memorial. This event brought together the crème de la crème of several generations of Moscow mathematicians (now scattered all around the world) whose life in mathematics was to a large extent shaped by I. M.'s influence. Despite the sad occasion, it was a pleasure to see many old friends and to share memories of our student years when we all attended the famous Gelfand Seminar at Moscow State University. And of course to share stories about I. M. He was such a huge presence in so many lives, and his passing left a gap which will be impossible to fill.

I first met I. M. in the early fall of 1970 . The meeting was arranged by Victor Gutenmacher, who at the time worked at the School by Correspondence organized by I. M. (with the purpose of bringing

Andrei Zelevinsky is professor of mathematics at Northeastern University. His email address is andrei@neu. edu.

Zelevinsky is grateful to his daughter, Katya, for helpful editorial suggestions. mathematics into the lives of schoolchildren all around the Soviet Union). I. M., who as usual was simultaneously involved in a myriad of various projects both scientific and pedagogical, had decided to organize and run a special mathematical class of seventh-graders within the famous Moscow School No. 2, which already had a decade-long tradition of such classes. To help him run this program, I. M. asked Victor to find him several young assistants who had themselves passed through such a class during their school years. I was very fortunate to become one of four such assistants. (Another was my old friend and classmate since the seventh grade Borya Feigin, who is now a distinguished mathematician.) All four of us had graduated a year earlier from the same School No. 2, all were math undergraduates beginning our second year at Moscow State University, and all felt a little lost in our mathematical studies.

The organizational matters could have been resolved within a few minutes, but our first meeting with I. M. lasted for several hours. The four of us (plus poor Victor) walked with I. M. for hours, and he talked to us about all kinds of things, mathematical and not. He asked us what we most loved about mathematics and what seminars and elective courses we had attended during our freshman year. Of course, he declared that we had done everything wrong and were almost lost for mathematics, but there was still some little hope for us if we started to attend his seminar at once. He explained to us how to study a new mathematical subject: focus on the most basic things at the foundation and dwell upon them until you reach full understanding; then the technicalities of the subject would be understood very quickly and effortlessly. I remember vividly how he illustrated this by explaining to us the foundations of linear algebra: a subspace in a vector space is characterized by one integer, its dimension; a pair of subspaces is characterized by three integers (dimensions of the two subspaces and of their intersection). What about a triple of subspaces? and what about a quadruple? ${ }^{6}$

\footnotetext{
${ }^{6}$ Triples of subspaces demonstrate the limitations of a fruitful analogy between subspaces of a finite-dimensional vector space and subsets of a finite set: the subspace lattice is only modular but not distributive. Thinking about this analogy led me to my first two published notes, which appeared within the next couple of years. As for the quadruples, as I
} 
This meeting was definitely one of my most lifechanging experiences. I have never met a person with such personal magnetism and such an ability to ignite enthusiasm about mathematics as I. M. I remember returning home late in the evening, totally exhausted but happy, with the definite feeling that my mathematical fate had been sealed that day. I could not sleep and spent most of the night thinking about mysteries of triples of subspaces! I attended the Gelfand Seminar for almost twenty years. A lot has been said about its unique character, including horror stories about I. M.'s rough treatment of speakers and participants. I had my share of humbling experiences there, both as a speaker and as a "control listener" who was sent by I. M. to the blackboard in the middle of a talk to explain what the speaker was trying to say. Many people, including some excellent mathematicians, could not stand this style and stopped attending the seminar. Those who stayed (myself included) decided that such a great learning experience was worth a little suffering. Equally important, or maybe even more important than the talks themselves, was Gelfand's choice of topics; his comments and monologues, which often deviated a lot from the original topic; and of course his famous "pedagogical" jokes and stories.

The official starting time of the seminar was 7 p.m. (or was it 6:30?) on Mondays, but it almost always started with much delay, sometimes after up to two hours! I am sure I. M. did this on purpose, because these weekly get-togethers before the seminar with numerous friends coming to the university from all around the city were also a big attraction. Sometimes even after his arrival, I. M. did not immediately start the seminar but stayed in the corridor for some time and chatted with people just like everybody else.

I think of myself as I. M.'s "mathematical grandson": my first real teacher and de facto Ph.D. advisor was Joseph Bernstein, one of Gelfand's best students. ${ }^{7}$ However, at some point after Joseph's emigration (in 1980, I believe), I. M. approached me and suggested that we start working together. Our close mathematical collaboration lasted about a decade (from the first joint note in 1984 to our

realized much later, I. M. had just finished his remarkable paper with V. A. Ponomarev, "Problems of linear algebra and classification of quadruples of subspaces in a finitedimensional vector space", so he was talking to us about his own cutting-edge research!

${ }^{7}$ Joseph could not be my official advisor, since he was never affiliated with the mathematics department at Moscow State, which was also the case for many other first-rate mathematicians from the "Gelfand circle". A. A. Kirillov Sr., also a student of Gelfand, kindly agreed to serve as my official advisor. Of course, I also learned a lot from him and from attending his seminar.
1994 book with Misha Kapranov). It would take volumes to give a comprehensive account of I. M.'s mathematical contributions, so let me just share my personal impressions of some of his unique features as a mathematician and as a teacher. ${ }^{8}$

Working with him could be a very frustrating experience. Just looking at his incredibly prolific scientific output, one would imagine him as a model of efficiency, never wasting a moment of his time. But most of the time during our daily meetings was filled with numerous distractions, jumps from one topic to another, his long phone conversations with an amazing variety of people on an amazing variety of topics, etc. Quite often after long hours spent like this I felt totally exhausted and had a depressing feeling that we had just wasted a perfectly good working day. But almost without exception there came a moment (sometimes when I was already saying good-bye at the door) of his total concentration on our project that led to extremely rapid progress, completely justifying all the torturous hours leading to this moment. It seemed as if his subconscious mind never stopped working on our project (and probably on a multitude of other things at the same time), and it just took I. M. a long time to become ready to spell out the results of this work.

This "nonlinearity" of I. M.'s thinking process was also one of the many features that made his seminar so unique. He would spend an inordinate amount of time asking everybody to explain to him some basic definitions and facts, and just when most of the participants (starting with the speaker, of course) would get totally frustrated, I. M. would suddenly switch gears and say something very illuminating, making it all worthwhile. ${ }^{9}$

I have never met any other mathematician with such an ability to see the "big picture" and always go to the heart of the matter, ignoring unnecessary technicalities. He had an uncanny ability to ask the "right" questions and to find unexpected connections between different mathematical fields. I. M. was fully aware of this gift and liked to illustrate it by one of his numerous "pedagogical" stories: "An old plumber comes to repair a heater. He goes around it, thinks for a moment, and hits it once with a hammer. The heater immediately starts working. The plumber charges 200 rubles for his service. The owner says, 'But you just spent two minutes and didn't do anything.' The plumber replies, 'I am charging you 3 rubles for hitting your

\footnotetext{
${ }^{8}$ For I. M. these two occupations were inseparable. His way of doing mathematics always involved close personal interaction with his innumerable students and collaborators.

${ }^{9}$ I. M. often interrupted speakers with the words "May I ask a stupid question?" The best reply to this was given by Y. I. Manin during one of his rare appearances as a speaker: "No, I. M., I don't think you are capable of such a thing!"
} 
heater with a hammer and the rest for knowing where to hit, which took me forty years to learn! ${ }^{10}$

I. M. had such an enormous wealth of ideas and plans that he needed many collaborators to help him realize even a small part of them. I was always very impressed with his great intuitive grasp of people. A few minutes of penetrating questioning of a new acquaintance allowed him to take the full measure of a person, understand his or her scientific potential, strengths and weaknesses, even how much pressure the person could withstand. In my own case, it seems that I. M. detected my soft spot for algebraic combinatorics (quite rare in Moscow at that time, I must say) even before I realized it myself. Very soon after I joined his seminar, he asked me to study (and then give a talk on) the old book The Theory of Group Characters and Matrix Representations of Groups by D. E. Littlewood, where the representation theory was treated with a strong combinatorial flavor. This was truly a sniper's shot: the facts and ideas that I learned from this book continue to serve me to this day. Another truly inspired suggestion by I. M. was to bring together Borya Feigin and Dmitry Borisovich Fuchs, which led to many years of very fruitful collaboration. Like so many of my friends and colleagues, I feel very fortunate for having known Israel Moiseevich and for being given a chance to be close to this gigantic, complex, wise, inspiring, and infinitely fascinating personality.

\footnotetext{
${ }^{10}$ I. M.'s amazing record of initiating new fruitful directions of mathematical research provides plenty of examples of "knowing where to hit." Let me just mention one example: the theory of general hypergeometric functions initiated by I. M. (where a significant part of my collaboration with him took place) grew from his insight that hypergeometric functions should live on the Grassmannians.
}

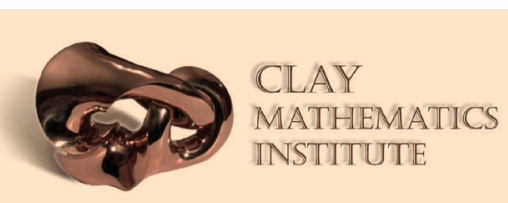

\section{Enhancement and Partnership Program}

The Clay Mathematics Institute invites proposals under its new program, "Enhancement and Partnership". The aim is to enhance activities that are already planned, particularly by funding international participation. The program is broadly defined, but subject to general principles:

- CMI funding will be used in accordance with the Institute's mission and its status as an operating foundation to enhance mathematical activities organised by or planned in partnership with other organisations.

- It will not be used to meet expenses that could be readily covered from local or national sources.

- All proposals will be judged by the CMI's Scientific Advisory Board.

Examples include:

- Funding a distinguished international speaker at a local or regional meeting.

- Partnership in the organisation of conferences and workshops.

- Funding a short visit by a distinguished mathematician to participate in a focused topical research program at an institute or university.

- Funding international participation in summer schools (lecturers and students) or repeating a successful summer school in another country.

- Funding a special lecture at a summer school or during a research institute program.

- Funding an extension of stay in the host country or neighbouring countries of a conference speaker.

Applications will only be received from institutions or from organisers of conferences, workshops, and summer schools. In particular the CMI will not consider applications under this program from individuals for funding to attend conferences or to visit other institutions or to support their personal research in other ways.

Enquiries about eligibility should be sent to president@ claymath.org. Applicants should set out in a brief letter a description of the planned activity, the way in which this could be enhanced by the CMI, the existing funding, the funds requested and the reason why they cannot be obtained from other local or national sources. Funds requested should not be out of proportion to those obtained from other sources. The CMI may request independent letters of support.

Applications should be sent to admin@claymath.org. There is no deadline, but the call will be closed when the current year's budget has been committed. 